\title{
Gauge-fixing, semiclassical approximation and potentials for graded Chern-Simons theories
}

\author{
C. I. Lazaroiu \\ C. N. Yang Institute for Theoretical Physics \\ SUNY at Stony Brook, NY11794-3840, U.S.A. \\ calin@insti.physics.sunysb.edu
}

\author{
R. Roiban \\ Department of Physics, UCSB \\ Santa Barbara, CA 93106, U.S.A. \\ radu@vulcan.physics.ucsb.edu
}

\begin{abstract}
We perform the Batalin-Vilkovisky analysis of gauge-fixing for graded Chern-Simons theories. Upon constructing an appropriate gauge-fixing fermion, we implement a Landau-type constraint, finding a simple form of the gauge-fixed action. This allows us to extract the associated Feynman rules taking into account the role of ghosts and antighosts. Our gauge-fixing procedure allows for zero-modes, hence is not limited to the acyclic case. We also discuss the semiclassical approximation and the effective potential for massless modes, thereby justifying some of our previous constructions in the Batalin-Vilkovisky approach.
\end{abstract}




\section{Contents}

1. Introduction $\quad 3$

2. Graded Chern-Simons theory 5

2.1 The set-up 5

$\begin{array}{lll}2.2 & \text { The action } & 7\end{array}$

$\begin{array}{lll}2.3 & \text { Hermitian structure } & 7\end{array}$

2.3.1 Metrics 7

2.3.2 The conjugation operator 8

2.3.3 The adjoint of $d$ and the Laplacian 8

2.4 Spacetime ghost grading 9

2.5 Dependence on the underlying superbundle 9

$\begin{array}{ll}\text { 3. The classical BV system } & 10\end{array}$

3.1 The tree-level master action $\quad 10$

3.2 Extended Hermitian product and conjugation 12

3.3 The extended Laplacian 13

4. Gauge fixing in the BV formalism $\quad 15$

4.1 Trivial pairs 16

4.2 A gauge-fermion of 'delta-function' type 18

4.2.1 Delta-function gauge-fixing in the absence of zero modes 19

4.2.2 Extension to the case with zero modes 23

4.3 Summary 26

$\begin{array}{ll}\text { 4.3.1 Cohomological formalism for } A_{\sigma} & 27\end{array}$

4.4 Simplified form of the gauge-fixed action 28

5. The partition function and semiclassical approximation 29

5.1 The semiclassical approximation 29

$\begin{array}{lll}5.1 .1 & \text { The first approach } & 30\end{array}$

5.1.2 The second approach 30

$\begin{array}{lll}5.1 .3 & \text { Regularization } & 31\end{array}$ 
6. The effective potential $\quad 32$

6.1 Perturbative expansion 32

6.2 The propagator of nonzero-modes 33

$\begin{array}{lll}6.3 & \text { Feynman rules } & 34\end{array}$

6.4 Tree-level products $\quad 35$

$\begin{array}{ll}\text { 7. Conclusions } & 36\end{array}$

$\begin{array}{ll}\text { A. A weighted gauge } & 37\end{array}$

$\begin{array}{lll}\text { A.1 The acyclic case } & 37\end{array}$

$\begin{array}{lll}\text { A.2 Inclusion of zero-modes } & 39\end{array}$

$\begin{array}{lll}\text { A.3 An example } & 40\end{array}$ 


\section{Introduction}

The study of D-brane composites is central to a better understanding of Calabi-Yau compactifications of open strings $[1,2,3]$. In a series of papers $[4,5,6,7,29,8,9]$ it was proposed that the topological counterpart of this problem (which can be formulated within the topological sigma model framework of $[11,12]$ ) can be studied with the tools of string field theory. This is a potentially fruitful approach, since it allows us to apply standard field theory techniques to a seemingly unrelated problem.

In particular, it was argued in [6] that the dynamics of A-type topological branes in the absence of worldsheet instantons can be described by a graded version of ChernSimons field theory, just as ungraded topological D-brane dynamics can be described in usual Chern-Simons language [10]. These models, whose equations of motion describe so-called 'flat superconnections of total degree one', seem to allow for a standard description of the extended moduli space of topological D-branes. From a purely field theoretic perspective, they form interesting generalizations of Chern-Simons field theory. It is therefore natural to ask how the results for the ungraded case extend to these more general systems.

In two recent papers $[8,9]$, we considered effective potentials and the semiclassical approximation for such models. Since graded Chern-Simons theories contain higher rank forms, a full justification of the gauge-fixing procedure used in those papers requires a detailed analysis in the Batalin-Vilkovisky formalism. The purpose of the present paper is to carry this out in full generality, thereby justifying certain points of $[8,9]$ and complementing the classical BV analysis already performed in [29], upon using the geometric formalism of $[13,14,15,16,17,18,19,20]$. This will be achieved by considering a Landau-type gauge, which leads to a simple expression for the gauge-fixed action reminiscent of the results of [21].

Since we wish to work generally, the resulting BV analysis is somewhat technical. Indeed, a general graded Chern-Simons theory contains fields of arbitrarily large ghost number, even though the form rank is constrained to lie in the interval $0 \ldots 3$. The reason for this is that the ghost degree results by shifting the form rank of the field through a quantity which depends on position of the corresponding block inside a matrix of bundle-valued forms. This has the effect of allowing an extended range of ghost numbers, which depends of the range of subbundle grades present in the system. To describe this situation, we will have to construct a gauge-fixing fermion which generally contains an arbitrary number of levels of extraghosts. This somewhat complicated structure can be described systematically upon using the general methods discussed in [22], and leads to a result which is reminiscent of the 'universal gauge fermion' considered in a different context in [23]. Despite the complexity of this construction, the 
result of gauge-fixing is surprisingly simple, and in many ways similar to the situation familiar from usual Chern-Simons theory. Indeed, our gauge fermion will implement a graded analogue of the standard Landau-gauge, and lead to a description of the gauge-fixed action which is formally similar to that used (for the ungraded case) in [21]. In fact, we shall be able to generalize even further, by including a convenient gauge-fixing procedure for zero modes (in particular, our construction is not limited to the acyclic case). For the Landau-type fermion, the zero-mode components depend on certain parameters described by positive operators defined on the harmonic subspace of various field, ghost and antighost configurations. This auxiliary data will be necessary in order to recover the description of the semiclassical approximation given in [9]. We also construct a family of weighted gauges, which reduces to the Landau gauge in an appropriate limit.

An interesting by-product of gauge-fixing is that it induces certain prefactors in the gauge-fixed path integral. These factors, which arise when integrating out the various auxiliary fields, are crucial for obtaining the correct path integral measure, and for recovering the results of [9] in the semiclassical approximation. In particular, we show that they are in complete agreement with the predictions of the method of resolvents $[24,25,26]$, which was used in [9]. In fact, we will be able to recover the results of [9] by a direct path integral computation. This agrees with the general observation $[27,28]$ that the method of resolvents is an indirect way of taking into account the role of ghosts and antighosts. Finally, we give a BV treatment of the effective potential for massless modes, thus justifying certain statements of [8].

The paper is organized as follows. In Section 2, we review the construction of graded Chern-Simons theories. We also discuss how one can introduce a Hermitian structure on the space of field configurations, upon using auxiliary metric data which will be required by our gauge-fixing procedure. In Section 3, we review the tree-level BV action for our systems, which was constructed in [6] and discussed in detail in [29]. We also explain how one can promote the Hermitian structure on the space of classical fields to the space of extended field configurations (which includes configurations of ghosts and antifields). Some of these issues have already been discussed in [6, 29] and [8], but we include a summary for reasons of completeness. In Section 4, we take up the problem of gauge-fixing in the BV formalism. Following standard procedure, we start in Subsection 4.1 by adding trivial pairs of extraghosts and auxiliary fields. Subsection 4.2. constructs the Landau gauge fixing fermion (a fermion of 'delta-function type') upon using the general methods explained in [22]. After describing the construction for both the harmonic and non-zero modes, we perform gauge-fixing by eliminating the antifields and integrating out the auxiliary fields. This leads to our gauge-fixed action, and produces certain prefactors in the gauge-fixed path integral. The result 
of the entire process is summarized in Subsection 4.3. The gauge-fixed action has a particularly simple form, which is reminiscent of that considered in [21] for the case of usual Chern-Simons theory. As explained in Subsection 4.4, this allows one to further simplify the gauge-fixed correlators, upon performing a certain change of variables for the antighosts. The result is a graded version of the formalism used in [21], further extended to take into account the zero-modes. In Section 5, we use our results to study the semiclassical approximation and the effective potential. Upon considering a background field configuration, we expand the action in quadratic and cubic terms, and separate the result into the Gaussian contribution (which is given entirely by the massive modes) and the contributions coming from cubic terms. Up to a prefactor induced by the gauge-fixing of zero-modes, the former gives the semiclassical partition function, which is computed by two equivalent methods in Subsection 5.1. Upon performing zeta-function regularization, the result is in complete agreement with that of [9], which was obtained by the method of resolvents discussed in [24, 25, 26, 30]. The higher contributions can be used to define an effective potential for the zero-modes, which was already studied in [8] with more elementary methods; this potential is induced due to the presence of interactions between harmonic and massive modes. In Section 6, we discuss the perturbative expansion of this potential, the relevant massive propagator and the tree-level approximation. For the latter, we explain the relation with the description of [8], and in particular justify the construction used in that paper from the BV perspective. Section 7 presents our conclusions. In the appendix, we show that the Landau-type gauge of the present paper can be obtained as a certain limit of a family of weighted gauges, a result which generalizes well-known constructions of standard gauge theory. While conceptually important, this result is not needed for understanding the rest of the paper. The appendix also discusses a simple example, showing how the weighted gauge produced by our general construction can be re-discovered through more elementary means.

\section{Graded Chern-Simons theory}

We start with a brief review of graded Chern-Simons theories on closed 3-manifolds. More details on their construction and basic properties can be found in [8, 29]. The relation with graded topological D-branes is explained in [6] (see also $[4,5])$.

\subsection{The set-up}

Consider an oriented closed (i.e. compact and boundary-less) 3-manifold $L$, and a (finite) collection of flat complex vector bundles $E_{n}$. We form the total bundle $\mathbf{E}=$ $\oplus_{n} E_{n}$, endowed with the $\mathbb{Z}$-grading induced by $n$. We shall make the convention that 
a form on $L$ of rank lying outside the interval $0 . .3$ is defined to be zero. Note that we consider complex flat vector bundles $E_{n}$, which are not required to be unitary (i.e. there need not exist metrics on $E_{n}$ which are covariantly-constant with respect to the flat connections).

The graded Chern-Simons theory of [6, 29] describes sections of the bundle:

$$
\mathcal{V}=\Lambda^{*}\left(T^{*} L\right) \otimes \operatorname{End}(\mathbf{E})
$$

which we endow with the total grading $\mathcal{V}=\oplus_{t} \mathcal{V}^{t}$, where:

$$
\mathcal{V}^{t}=\bigoplus_{\substack{k, m, n \\ k+n-m=t}} \Lambda^{k}\left(T^{*} L\right) \otimes \operatorname{Hom}\left(E_{m}, E_{n}\right)
$$

The space of sections $\mathcal{H}=\Gamma(\mathcal{V})=\Omega^{*}(L, \operatorname{End}(\mathbf{E}))$ is endowed with the grading $\mathcal{H}^{k}=$ $\Gamma\left(\mathcal{V}^{k}\right)$. The degree of a section $u \in \mathcal{H}$ has the form:

$$
|u|=r k u+\Delta(u)
$$

where $\Delta(u)=n-m$ if $u \in \Omega^{*}\left(L, \operatorname{Hom}\left(E_{m}, E_{n}\right)\right)$.

The action is most conveniently described in terms of the the so-called total boundary product:

$$
u \bullet v=(-1)^{\Delta(u) r k v} u \wedge v
$$

where the wedge product is understood to include composition of bundle morphisms. This associative product has the properties:

$$
|u \bullet v|=|u|+|v|, \quad 1 \bullet u=u \bullet 1=u,|u|=0,
$$

where 1 stands for the identity endomorphism of $\mathbf{E}$.

The direct sum $A^{(0)}=\oplus_{n} A_{n}$ of the flat connections carried by $E_{n}$ induces a flat structure on $\operatorname{End}(\mathbf{E})$, and a differential $d^{(0)}$ (the de Rham differential twisted by this flat connection), which acts as a degree one derivation of the boundary product. More general backgrounds are obtained upon shifting by degree one elements $\phi$ of $\mathcal{H}$, which leads to the shifted differential $d=d^{(0)}+[\phi, .]_{\bullet}$, where $[., .]_{\bullet}$ stands for the graded commutator:

$$
[u, v]_{\bullet}:=u \bullet v-(-1)^{|u||v|} v \bullet u
$$

Then $d$ is a degree one derivation of the associative algebra $(\mathcal{H}, \bullet)$ :

$$
|d u|=|u|+1, \quad d(u \bullet v)=(d u) \bullet v+(-1)^{|u|} u \bullet(d v) .
$$

In the language of [31], $d$ defines 'graded superconnection [32] of total degree one'. For what follows, we pick a reference background $\phi$ satisfying $d^{2}=0$. 
To write down the action, we also introduce the graded trace of elements $u$ in $\mathcal{H}$ :

$$
\operatorname{str}(u)=\sum_{n}(-1)^{n} \operatorname{tr}\left(u_{n n}\right), \text { for } u=\oplus_{m, n} u_{m n},
$$

with $u_{m n} \in \Omega^{*}\left(L, \operatorname{Hom}\left(E_{m}, E_{n}\right)\right)$. This allows us to define the nondegenerate bilinear form:

$$
\langle u, v\rangle:=\int_{L} \operatorname{str}(u \bullet v)
$$

which has the properties:

$$
\langle u, v\rangle=(-1)^{|u||v|}\langle v, u\rangle,\langle d u, v\rangle+(-1)^{|u|}\langle u, d v\rangle=0 \quad, \quad\langle u \bullet v, w\rangle=\langle u, v \bullet w\rangle(2.10)
$$

and obeys the selection rule $\langle u, v\rangle=0$ unless $|u|+|v|=3$.

\subsection{The action}

The graded Chern-Simons theory is described by the action:

$$
S(\phi)=\int_{L} \operatorname{str}\left[\frac{1}{2} \phi \bullet d \phi+\frac{1}{3} \phi \bullet \phi \bullet \phi\right]+c c=\frac{1}{2}\langle\phi, d \phi\rangle+\frac{1}{3}\langle\phi, \phi \bullet \phi\rangle+c c \quad,
$$

which is defined on the degree one subspace:

$$
\mathcal{H}^{1}=\{\phi \in \mathcal{H}|| \phi \mid=1\}=\Gamma\left(\oplus_{k+n-m=1} \Lambda^{k}\left(T^{*} L\right) \otimes \operatorname{Hom}\left(E_{m}, E_{n}\right)\right)
$$

The equations of motion have the form:

$$
d \phi+\frac{1}{2}[\phi, \phi] \bullet=0 \Leftrightarrow d \phi+\phi \bullet \phi=0 \quad\left(\phi \in \mathcal{H}^{1}\right)
$$

and are equivalent with the requirement that the shifted superconnection $d_{\phi}=d+[\phi,.] \bullet$ is flat (i.e. satisfies $\left(d_{\phi}\right)^{2}=0$ ). These equations are invariant under transformations of the form:

$$
\phi \rightarrow \phi^{g}=g \bullet \phi \bullet g^{-1}+g \bullet d g^{-1},
$$

where $g$ is an invertible element of the subalgebra $\left(\mathcal{H}^{0}, \bullet\right)$. Dividing the space of solutions to (2.13) through these symmetries gives a moduli space $\mathcal{M}$.

\subsection{Hermitian structure}

\subsubsection{Metrics}

The gauge-fixing procedure discussed in Section 4 will require choosing a Riemannian metric $g$ on $L$ and Hermitian metrics on the bundles $E_{n}$. These a Hermitian metric $g_{\mathbf{E}}$ on $\mathbf{E}$, and thus a Hermitian metric on $\operatorname{End}(\mathbf{E})$ :

$$
(\alpha, \beta)=\operatorname{tr}\left(\alpha^{\dagger} \circ \beta\right) \quad \text { for } \quad \alpha, \beta \in \operatorname{End}\left(\mathbf{E}_{p}\right), p \in L
$$


where $\alpha^{\dagger}$ is the Hermitian conjugate of $\alpha$ with respect to $g_{\mathbf{E}}$. On the other hand, $g$ induces a Hermitian metric $(.,$.$) on \Lambda^{*}\left(T^{*} L\right)$ :

$$
(* \bar{\omega}) \wedge \eta=(\omega, \eta) v o l_{g} \quad, \text { for } \quad \omega, \eta \in \Lambda^{*}\left(T_{p}^{*} L\right)
$$

where $\mathrm{vol}_{g}$ is the volume form induced by $g$ on $L$ (with respect to the orientation on $L$ ), while $*$ is the complex linear Hodge operator, which satisfies:

$$
r k(* \omega)=3-r k \omega \quad \text { and } \quad *^{2}=i d
$$

Combining everything, we obtain a Hermitian metric $(.,.) \mathcal{V}$ on the bundle $\mathcal{V}=\Lambda^{*}\left(T^{*} L\right) \otimes$ $\operatorname{End}(\mathbf{E})$ :

$$
\operatorname{tr}\left(* u^{\dagger} \wedge v\right)=(u, v)_{\mathcal{V}} v o l_{g} \quad \text { for } u, v \in \mathcal{V}_{p} \quad, \quad p \in L
$$

Integration over $L$ gives a Hermitian scalar product on the space $\mathcal{H}=\Gamma(\mathcal{V})$ :

$$
h(u, v)=\int_{L}(u, v) v o l_{g}=\int_{L} \operatorname{tr}\left(* u^{\dagger} \wedge v\right), \text { for } u, v \in \mathcal{H} .
$$

\subsubsection{The conjugation operator}

Since the bilinear form (2.9) is non-degenerate, there exists a unique antilinear operator $c$ on $\mathcal{H}$ with the property:

$$
h(u, v)=\langle c u, v\rangle=\int_{L} \operatorname{str}[(c u) \bullet v] .
$$

This has the following form on decomposable elements:

$c(\omega \otimes f)=(-1)^{n+\Delta(f)(1+r k \omega)}(* \bar{\omega}) \otimes f^{\dagger} \quad, \quad$ for $\quad \omega \in \Omega^{*}(L) \quad$ and $\quad f \in \operatorname{Hom}\left(E_{m}, E_{n}\right)$

and satisfies:

$$
|c u|=3-|u|, \quad c^{2}=i d, \quad h(u, v)=h(c v, c u)
$$

(in particular, $c$ is anti-unitary). In the ungraded case $\left(\mathbf{E}=E_{0}\right), c$ reduces to the antilinear Hodge operator $₹$, coupled to the bundle End $(E)$.

\subsubsection{The adjoint of $d$ and the Laplacian}

The scalar product $h$ satisfies the selection rule:

$$
h(u, v)=0, \quad \text { unless } \quad|u|=|v| .
$$

Considering the Hermitian conjugate $d^{\dagger}$ of $d$ (with respect to $h$ ), one has:

$$
d^{\dagger} u=(-1)^{|u|} c d c u, \quad\left|d^{\dagger} u\right|=|u|-1, \quad\left(d^{\dagger}\right)^{2}=0 \quad, \quad\left\langle d^{\dagger} u, v\right\rangle=(-1)^{|u|}\left\langle u, d^{\dagger} v\right\rangle .
$$


and:

$$
\begin{aligned}
& c d^{\dagger} u=(-1)^{|u|} d c u, \quad d^{\dagger} c u=(-1)^{|u|+1} c d u \\
& d^{\dagger} d c=c d d^{\dagger} \quad, \quad d d^{\dagger} c=c d^{\dagger} d .
\end{aligned}
$$

One also constructs the 'deformed Laplacian' $\Delta=d d^{\dagger}+d^{\dagger} d$, which will be useful below.

We recall the Hodge decompositions:

$$
\mathcal{H}=K \oplus i m d \oplus i m d^{\dagger} \quad, \quad \text { kerd }=i m d \oplus K \quad, \quad k e r d^{\dagger}=i m d^{\dagger} \oplus K \quad,
$$

where $K=\operatorname{ker} \Delta=\operatorname{ker} d \cap k e r d^{\dagger}$. It will be useful to consider the orthogonal projectors $\pi_{d}=d \frac{1}{\Delta} d^{\dagger}, \pi_{d^{\dagger}}=d^{\dagger} \frac{1}{\Delta} d$ and $P=1-\pi_{d}-\pi_{d^{\dagger}}$ of $\mathcal{H}$ on the subspaces $i m d, i m d^{\dagger}$ and $K$, as well as the propagator $U:=\frac{1}{d} \pi_{d}$. The first of equations (2.25) implies the relation:

$$
\pi_{d} c=c \pi_{d^{\dagger}}
$$

\subsection{Spacetime ghost grading}

For what follows, it will be convenient to use the modified grading $s(u)=1-|u|$, which in the BV formalism corresponds to the ghost number of the string field theory [29]. We shall use the notation $\mathcal{H}(\sigma)=\mathcal{H}^{1-\sigma}$ for the homogeneous subspaces of $\mathcal{H}$ with respect to this grading, and the notation $H_{\sigma}(\mathcal{H})=H^{1-\sigma}(\mathcal{H})$ for the associated components of $H^{*}(\mathcal{H})$ (note that ghost grading is decreased by $d$, which justifies the homological notation). With this convention, the string field lies in the subspace $\mathcal{H}(0)$. One has:

$$
s(d u)=s(u)-1, \quad s(c u)=-1-s(u), \quad s(c d u)=-s(u) .
$$

In particular, $c d$ induces an antilinear operator on the physical subspace $\mathcal{H}(0)$.

\subsection{Dependence on the underlying superbundle}

It is instructive to consider to what extend the various data are specified by the superbundle underlying our graded bundle. For this, we define subbundles:

$$
E_{\text {even }}=\oplus_{n=\text { even }} E_{n} \text { and } E_{\text {odd }}:=\oplus_{n=o d d} E_{n}
$$

so that $\mathbf{E}=E_{\text {even }} \oplus E_{\text {odd }}$. Viewed in this manner, $\mathbf{E}$ becomes a superbundle $\left(=\mathbb{Z}_{2^{-}}\right.$ graded bundle), if one forgets the finer $\mathbb{Z}$-grading given by the decompositions of $E_{\text {even }}$ and $E_{\text {odd }}$. Accordingly, the bundle $\mathcal{V}$ only remembers the $\mathbb{Z}_{2}$-grading $\mathcal{V}=\mathcal{V}^{\text {even }} \oplus \mathcal{V}^{\text {odd }}$, where:

$$
\mathcal{V}^{\text {even }}:=\oplus_{k=\text { even }} \mathcal{V}^{k} \text { and } \mathcal{V}^{\text {odd }}:=\oplus_{k=\text { odd }} \mathcal{V}^{k}
$$


These subbundles only depend on the superbundle structure of $\mathbf{E}$ :

$$
\begin{array}{r}
\mathcal{V}^{\text {even }}=\oplus_{j=\text { even }}\left[\Omega^{j}\left(L, \operatorname{Hom}\left(E_{\text {even }}, E_{\text {even }}\right)\right) \oplus \Omega^{j}\left(L, \operatorname{Hom}\left(E_{\text {odd }}, E_{\text {odd }}\right)\right)\right] \oplus \\
\oplus_{j=\text { odd }}\left[\Omega^{j}\left(L, \operatorname{Hom}\left(E_{\text {even }}, E_{\text {odd }}\right)\right) \oplus \Omega^{j}\left(L, \operatorname{Hom}\left(E_{\text {odd }}, E_{\text {even }}\right)\right)\right],
\end{array}
$$

and a similar relation for $\mathcal{V}^{\text {odd }}$. Moreover, the $\mathbb{Z}$-grading on $\mathcal{H}$ induces a $\mathbb{Z}_{2}$-grading which is only sensitive to the superbundle structure of $\mathbf{E}$ :

$$
\mathcal{H}^{\text {even }}:=\oplus_{k=\text { even }} \mathcal{H}^{k}=\Gamma\left(\mathcal{V}^{\text {even }}\right), \quad \mathcal{H}^{\text {odd }}:=\oplus_{k=\text { odd }} \mathcal{H}^{k}=\Gamma\left(\mathcal{V}^{\text {odd }}\right) \text {. }
$$

With respect to this $\mathbb{Z}_{2}$-grading, the boundary algebra $(\mathcal{H}, d, \bullet)$ becomes a differential superalgebra. The essential point, however, is that this description does not suffice in order to identify the physical fields, and in particular does not uniquely specify the physical theory. Indeed, the physical subspace $\mathcal{H}^{1}=\{\phi \in \mathcal{H}|| \phi \mid=1\}$ knows about the $\mathbb{Z}$-grading of $\mathbf{E}$. The same is true about the gauge-group (2.14), whose structure depends markedly of this grading. Therefore, the physical content of the theory is entirely different for various choices of $\mathbb{Z}$-grading compatible with a given $\mathbb{Z}_{2}$-grading. This becomes especially clear when one considers the moduli space, whose structure is very sensitive to choice of a $\mathbb{Z}$-valued grading. Accordingly, the nature of the fields one can condense depends on the precise choice of ghost grading, since it is this data which specifies which fields are physical. We also stress that our theories are quite different from the super-Chern-Simons theories of $[34,33]$. In fact, the latter only contain physical one-form fields, while our theories will generally contain physical fields of rank different from one when both $E^{\text {even }}$ and $E^{o d d}$ are non-vanishing. It is precisely for this reason that condensation of fields which are not one-forms can be achieved in our framework, thereby allowing for an interpretation in terms of topological D-branes [6].

\section{The classical BV system}

\subsection{The tree-level master action}

Let us begin by recalling some results ${ }^{1}$ of [29], which will necessary below. Since the gauge algebra of (2.11) is generally reducible, a proper formulation of our theories requires the BV formalism already at the classical level. The corresponding classical master action was constructed in [6, 29]. The conclusion is as follows. The tree-level BV action associated with (2.11) is given by:

$$
S_{e}(\hat{\phi})=2 \operatorname{Re} \int_{L} \operatorname{str}_{e}\left[\frac{1}{2} \hat{\phi} * d \hat{\phi}+\frac{1}{3} \hat{\phi} * \hat{\phi} * \hat{\phi}\right]=\operatorname{Re}\left[\langle\hat{\phi}, d \hat{\phi}\rangle_{e}+\frac{2}{3}\langle\hat{\phi}, \hat{\phi} * \hat{\phi}\rangle_{e}\right],
$$

\footnotetext{
${ }^{1}$ The construction presented here (following [29]) is carried out within the framework of [36]. An alternate (and possibly better) point of view is the Berezin theory of superschemes [37]. We prefer to use the formulation of $[36,38]$ due to its being better established in the physics literature.
} 
where the extended field $\hat{\phi}$ is an element of the subspace $M:=\mathcal{H}_{e}^{1}=\left\{\hat{\phi} \in \mathcal{H}_{e} \mid \operatorname{deg} \hat{\phi}=\hat{1}\right\}$ of the so-called extended boundary space $\mathcal{H}_{e}$. The latter is constructed as the tensor product:

$$
\mathcal{H}_{e}=\mathcal{H} \otimes G
$$

where $G$ is a (complex) auxiliary Grassmann algebra. As in [29], we use $g$ to denote the $\mathbb{Z}_{2}$-degree on $G$ and deg to denote the induced $\mathbb{Z}_{2}$-valued degree on $\mathcal{H}_{e}$ :

$$
\operatorname{deg}(u \otimes \alpha)=|u|(\bmod 2)+g(\alpha)
$$

where $u \in \mathcal{H}$ and $\alpha \in G$. When endowed with this grading, the extended boundary space is a differential superalgebra with respect to the extended boundary product * defined through:

$$
(u \otimes \alpha) *(v \otimes \beta)=(-1)^{|v| g(\alpha)}(u \bullet v) \otimes(\alpha \beta)
$$

and the differential $d_{e}=d \otimes i d_{G}$. We also extend the gradings $|$.$| and g$ to partial gradings on $\mathcal{H}_{e}$ by:

$$
|u \otimes \alpha|:=|u| \text { and } g(u \otimes \alpha):=g(\alpha),
$$

so that deg$\hat{u}=|\hat{u}|(\bmod 2)+g(\hat{u})$ for all $\hat{u}$ in $\mathcal{H}_{e}$.

The $G$-valued, complex-bilinear form $\langle., .\rangle_{e}$ appearing in (3.1) is defined through (for decomposable elements $\hat{u}=u \otimes \alpha$ and $\hat{v}=v \otimes \beta$ ):

$$
\langle u \otimes \alpha, v \otimes \beta\rangle_{e}=(-1)^{|v| g(\alpha)}\langle u, v\rangle \alpha \beta=\int_{L} \operatorname{str}_{e}(\hat{u} * \hat{v})
$$

with the extended supertrace given by:

$$
\operatorname{str}_{e}(\omega \otimes f \otimes \alpha)=\operatorname{str}(f) \omega \otimes \alpha
$$

for $\omega \in \Omega^{*}(L), f \in \operatorname{End}(\mathbf{E})$ and $\alpha \in G$.

The ghost grading of the BV formalism is given by:

$$
s(\hat{u}):=1-|\hat{u}|
$$

and leads to a decomposition $\mathcal{H}_{e}=\oplus_{\sigma} \mathcal{H}_{e}(\sigma)$, where $\mathcal{H}_{e}(\sigma):=\mathcal{H}^{1-\sigma} \otimes G$ is the subspace of elements of ghost number equal to $\sigma$. Accordingly, the odd subspace $M=\mathcal{H}_{e}^{1}$ decomposes as:

$$
M=\oplus_{\sigma} M(\sigma), \text { with } M(\sigma)=\mathcal{H}_{e}^{1} \cap \mathcal{H}_{e}(\sigma)=\left\{\hat{\phi} \in \mathcal{H}_{e} \mid s(\hat{\phi})=\sigma \quad \text { and } \operatorname{deg} \hat{\phi}=\hat{1}\right\}
$$


The extended field has the decomposition:

$$
\hat{\phi}=\oplus_{\sigma} \hat{\phi}_{\sigma}=\oplus_{\sigma \geq 0} \phi_{\sigma}^{*} \oplus \oplus_{\sigma \geq 0} \phi_{\sigma} \quad, \quad \text { with } s\left(\hat{\phi}_{\sigma}\right)=\sigma \Leftrightarrow \hat{\phi}_{\sigma} \in M(\sigma)
$$

where we introduced the notations $\hat{\phi}_{\sigma}=\phi_{\sigma}$ for $\sigma \geq 0$ and $\hat{\phi}_{\sigma}=\phi_{-1-\sigma}^{*}$ for $\sigma<0$. The component $\hat{\phi}_{0}=\phi_{0} \in M(0)$ is the classical field, related to the unextended field $\phi$ of Section 2 through:

$$
\tilde{e} v_{G}\left(\phi_{0}\right)=\phi
$$

where $\tilde{e} v_{G}:=i d \otimes e v_{G}: \mathcal{H}_{e} \rightarrow \mathcal{H}$ is the extension of the obvious evaluation map $e v_{G}: G \rightarrow \mathbf{C}$. One has $\tilde{e} v_{G}(M(0))=\mathcal{H}^{1}$. The components $\phi_{\sigma}(\sigma>0)$ play the role of ghosts, while $\phi_{\sigma}^{*}(\sigma \geq 0)$ are the antifields. It will aso be convenient to describe the collections of fields and antifields by the elements $\hat{\phi}_{+}:=\bigoplus_{\sigma \geq 0} \hat{\phi}_{\sigma}$ and $\hat{\phi}_{-}:=\oplus_{\sigma<0} \hat{\phi}_{\sigma}$, such that $\hat{\phi}:=\hat{\phi}_{-} \oplus \hat{\phi}_{+}$.

The $G$-valued extended action (3.1) relates to the classical action (2.11) as follows (figure 1):

$$
e v_{G}\left(S_{e}(\hat{\phi})\right)=S\left(\tilde{e} v_{G}(\hat{\phi})\right) \text { for } \hat{\phi} \in M(0):=\left\{\hat{\phi} \in \mathcal{H}_{e}^{1} \| \hat{\phi} \mid=1\right\}
$$

The restriction of $*$ to the subspace $M(0)$ coincides with the unextended product $\bullet$ up to application of $\tilde{e} v_{G}$ :

$$
\tilde{e} v_{G}(\hat{u} * \hat{v})=\tilde{e} v_{G}(u) \bullet \tilde{e} v_{G}(v) \quad, \quad \text { for } \quad \hat{u} \text { and } \hat{v} \in M(0)
$$

We refer the reader to [29] for more details on the extended boundary data and the associated BV system.

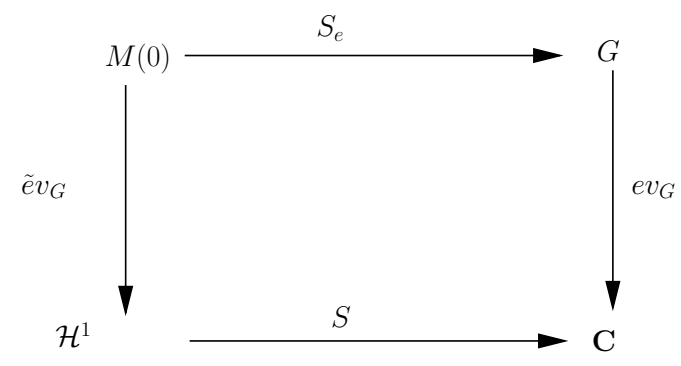

Figure 1: Relation between the extended and unextended actions.

\subsection{Extended Hermitian product and conjugation}

As in Subsection 2.2., let us pick metrics on $L$ and $E_{n}$. We wish to extend the Hermitian data of that section to the space $\mathcal{H}_{e}$. For this, we shall assume that the Grassmann 
algebra $G$ is endowed with a complex conjugation, i.e. a complex-antilinear, involutive operator $: G \rightarrow G$ which satisfies:

$$
\overline{\alpha \beta}=\bar{\beta} \bar{\alpha}, g(\bar{\alpha})=g(\alpha)
$$

This allows us to define the extended conjugation operator $c_{e}: \mathcal{H}_{e} \rightarrow \mathcal{H}_{e}$ via:

$$
c_{e}(u \otimes \alpha)=(-1)^{g(\alpha)(|u|+1)} c(u) \otimes \bar{\alpha} .
$$

It is clear that $c_{e}$ is complex-antilinear and satisfies:

$$
\left|c_{e}(\hat{u})\right|=3-|\hat{u}| \quad, \quad g\left(c_{e}(\hat{u})\right)=g(\hat{u}) \quad, \quad \operatorname{deg} c_{e}(\hat{u})=\hat{1}+\operatorname{deg} \hat{u} .
$$

A simple computation shows that:

$$
c_{e}^{2}(\hat{u})=(-1)^{g(\hat{u})} \hat{u}
$$

We also define an extended Hermitian product through:

$$
h_{e}(u \otimes \alpha, v \otimes \beta)=h(u, v) \bar{\alpha} \beta .
$$

This Grassmann-valued pairing has the property:

$$
\overline{h_{e}(\hat{u}, \hat{v})}=h_{e}(\hat{v}, \hat{u})
$$

Moreover, it is easy to check that:

$$
h_{e}(\hat{u}, \hat{v})=\left\langle c_{e} \hat{u}, \hat{v}\right\rangle_{e}
$$

which parallels the defining relation of the unextended conjugation $c$. The hermicity property (3.19) also reads

$$
\left\langle c_{e} \hat{u}, \hat{v}\right\rangle_{e}=\overline{\left\langle c_{e} \hat{v}, \hat{u}\right\rangle_{e}}
$$

a relation which will be useful below.

\subsection{The extended Laplacian}

If we define $d_{e}^{\dagger}=d^{\dagger} \otimes i d_{G}$, then a simple computation gives:

$$
d_{e}^{\dagger} \hat{u}=(-1)^{|\hat{u}|} c_{e} d_{e} c_{e} \hat{u} \quad, \quad h_{e}\left(\hat{u}, d_{e} \hat{v}\right)=h_{e}\left(d_{e}^{\dagger} \hat{u}, \hat{v}\right) .
$$

The extended Laplacian $\Delta_{e}=d_{e}^{\dagger} d_{e}+d_{e} d_{e}^{\dagger}=\Delta \otimes i d_{G}$ is Hermitian in the following sense:

$$
h_{e}\left(\Delta_{e} \hat{u}, \hat{v}\right)=h_{e}\left(\hat{u}, \Delta_{e} \hat{v}\right)
$$


It is easy to check that the last equation of (2.24) implies:

$$
\left\langle d_{e}^{\dagger} \hat{u}, \hat{v}\right\rangle_{e}=(-1)^{\operatorname{deg} \hat{u}}\left\langle\hat{u}, d_{e}^{\dagger} \hat{v}\right\rangle_{e}
$$

One can also check the relation:

$$
\left\langle\hat{u}, d_{e} c_{e} \hat{v}\right\rangle_{e}=(-1)^{(\operatorname{deg} \hat{u}+1) \operatorname{deg} \hat{v}} \overline{\left\langle c_{e} d_{e} \hat{u}, \hat{v}\right\rangle_{e}},
$$

which will be useful in Section 4 .

As in Subsection 2.2.2, we consider the orthogonal projectors $\pi_{d_{e}}=d_{e} \frac{1}{\Delta_{e}} d_{e}^{\dagger}=$ $\pi_{d} \otimes i d_{G}, \pi_{d_{e}^{\dagger}}=d_{e}^{\dagger} \frac{1}{\Delta_{e}} d_{e}=\pi_{d^{\dagger}} \otimes i d_{G}$ and $P_{e}=1-\pi_{d_{e}}-\pi_{d_{e}^{\dagger}}=P \otimes i d_{G}$ of $\mathcal{H}_{e}$ on the subspaces $i m d_{e}=(i m d) \otimes G, i m d_{e}^{\dagger}=\left(i m d^{\dagger}\right) \otimes G$ and $K_{e}:=\operatorname{kerd}_{e} \cap \operatorname{kerd}_{e}^{\dagger}=K \otimes G$. We have:

$$
\begin{array}{cl}
c_{e} d_{e}^{\dagger} \hat{u}=(-1)^{\operatorname{deg} \hat{u}} d_{e} c_{e} \hat{u} \quad, \quad d_{e}^{\dagger} c_{e} \hat{u}=(-1)^{1+\operatorname{deg} u} c_{e} d_{e} \hat{u} \\
d_{e}^{\dagger} d_{e} c_{e}=c_{e} d_{e} d_{e}^{\dagger} \quad, \quad d_{e} d_{e}^{\dagger} c_{e}=c_{e} d_{e}^{\dagger} d_{e}
\end{array}
$$

which generalizes equations (2.25). The first relations in (3.26) imply:

$$
c_{e} \pi_{d_{e}^{\dagger}}=\pi_{d_{e}} c_{e}
$$

We further note that the invertible operator $c_{e}$ maps $i m d_{e}^{\dagger}$ into $i m d_{e}$ and viceversa. As in [35], $d_{e}^{\dagger}$ gives a bijection between $i m d_{e}$ and $i m d_{e}^{\dagger}$, which means that $c_{e} d_{e}$ is invertible as an operator form $i m d_{e}^{\dagger}$ to itself.

We end with an observation which will be useful in the next section. If $f$ is a $G$-valued function, then we define its 'real part' by $R e f:=\frac{1}{2}(f+\bar{f})$. Ref is $G$-valued and has the property $\overline{R e f}=R e f=R e \bar{f}$. If $\hat{v}$ is a fixed element of $\mathcal{H}_{e}$, then the linear functional:

$$
\eta_{\hat{v}}(\hat{u}):=\operatorname{Re}\langle\hat{v}, \hat{u}\rangle_{e}
$$

is differentiable at the origin, with differential:

$$
d_{0} \eta_{\hat{v}}(\hat{w})=\operatorname{Re}\langle\hat{v}, \hat{w}\rangle_{e}
$$

It is clear that $d_{e} \eta_{v}$ vanishes if and only if $\langle\hat{w}, \hat{v}\rangle_{e}=0$ for all $\hat{w} \in \mathcal{H}_{e}$; this follows upon substituting $\hat{w}$ by $i \hat{w}$ and considering both equations. Since the unextended bilinear form is non-degenerate, this implies vanishing of $\hat{v}$.

If $F$ is a $G$-valued functional of $\hat{u} \in \mathcal{H}_{e}$, then we define the (generally non-linear) operator $\frac{\delta F}{\delta \hat{u}}: \mathcal{H}_{e} \rightarrow \mathcal{H}_{e}$ by:

$$
\delta F(\delta \hat{w})=\left\langle\frac{\delta F}{\delta \hat{u}}(\hat{u}), \delta \hat{w}\right\rangle_{e}=\operatorname{Re}\left\langle\frac{\delta F}{\delta \hat{u}}(\hat{u}), \delta \hat{w}\right\rangle_{e}
$$

where we used the fact that $\delta F(\delta \hat{w})=\operatorname{Re} \delta F(\delta \hat{w})$. With this definition, one has $\frac{\delta \eta_{v}}{\delta \hat{u}}=\hat{v}$, which is constant on $\mathcal{H}_{e}$. 


\section{Gauge fixing in the BV formalism}

We are now ready to perform the BV analysis of gauge-fixing. This will serve as justification for the effective potential discussed in [8] and completes the classical BV treatment of [29]. It will also allow us to recover the semiclassical approximation of [9] through a purely path integral approach. As a by-product, we shall find an invariant expression for the propagators of physical fields and ghost/antighosts. While our description entails a certain level of abstraction, we shall be able to give an entirely general discussion of graded Chern-Simons systems, which is valid for an arbitrary number of flat bundles $E_{n}$. Since the next two subsections are somewhat technical, the casual reader can read this introduction and jump directly to Subsection 4.3, which summarizes the results. The contents of that subsection suffice for understanding the rest of the paper.

Before proceeding with the technical details, let us explain the main points of our procedure. We wish to fix a background flat superconnection (i.e. a solution of the equations of motion) and build a local description of our theory around that background. In general, the background superconnection will not be isolated. Let $d$ denotes the differential of Section 2 in such a background (note that $d$ does not arise from the original flat connections, but it is 'twisted' with the background superconnection, in spite of our simplified notations). Then the linearized approximation to the moduli problem $(2.13,2.14)$ shows that infinitesimal deformations of the background are described (in first approximation) by the cohomology space $H_{d}^{1}(\mathcal{H})$; this description will be corrected at higher orders due the fact that some linearized deformations will be obstructed, so the space $H_{d}^{1}(\mathcal{H})$ describes virtual moduli (obstructions will be described by the effective potential, as in [8]). If the first cohomology of $d$ does not vanish, then one has to perform the path integral in the presence of zero modes. The standard description in this situation is to separate massive fluctuations around the background, and to decompose the path integral into an ordinary integral over zero modes (i.e. over the moduli space) and a path integral over the massive modes. Since we are interested in a local description, we shall proceed in a slightly different manner. Namely, we shall treat the zero modes in the linearized approximation (i.e. as virtual moduli), while treating the nonzero-modes exactly. The effect of this will be to produce a potential for the zero modes (obtained by integrating out the massive fluctuations), which in turn allows one to describe obstructions to infinitesimal deformations, and thus give an equivalent local formulation of the moduli problem [35, 8].

Since we do not wish to integrate over (virtual) zero modes, we shall pick a gaugefixing procedure which freezes these to some particular values. More precisely, we shall

pick auxiliary metrics and describe elements in $H_{d}^{1}(\mathcal{H})$ by the harmonic component 
$\phi^{K}$ of the physical field $\phi$, upon using the Hodge-theoretic decomposition discussed in Section 2. Our gauge-fixing fermion will freeze the virtual zero mode $\phi^{K}$ to some value $\phi^{H}$, which plays the role of a parameter in the gauge fermion. We shall allow nonzero values of $\phi^{H}$, since we wish to build an effective potential for the virtual zero modes, hence we must allow them to take different values. In fact, $\phi^{H}$ should be viewed as an (infinitesimal) potential deformation of the background superconnection - which will be obstructed or not, depending on whether it lies along a valley of the effective potential or fails to be contained in its critical set.

This basic picture must of course be modified by taking into account the ghosts and antighosts required for fixing the gauge symmetries of the original action. Since these are partners of the various components of the physical field, we shall treat them in a similar manner. Namely, we decompose all ghosts and antighosts into harmonic (massless) and nonharmonic (massive) components, and treat them separately in the gauge-fixing fermion. As for the physical field, the massless ghost/antighost modes will be fixed to some arbitrary values (we shall later take these to be zero, so that only the physical harmonic mode $\phi^{H}$ survives as a non-vanishing parameter). For the massive modes, we pick a Landau gauge condition, which reproduces the Lorentz gauge used in [8]. This will be implemented by a 'universal' gauge-fixing fermion of deltafunction type, built according to the general rules explained in [22]. Its construction is performed in standard manner, after adding an appropriate collection of trivial pairs to the original set of fields and antifields.

\subsection{Trivial pairs}

To perform gauge-fixing of the BV action (3.1), we must introduce an appropriate number of trivial pairs and pick a convenient gauge-fixing fermion [22]. Since we wish to do this in general, it will prove convenient to use the following notation.

Let $\phi[0]=\hat{\phi}^{+}=\oplus_{\sigma \geq 0} \phi_{\sigma}$ and $\phi^{*}[0]=\hat{\phi}_{-}=\oplus_{\sigma \geq 0} \phi_{\sigma}^{*}$ be the original collections of $\mathrm{BV}$ fields and antifields. We introduce new fields $\phi[k]=\oplus_{\sigma \geq 2 k} \phi_{\sigma}[k] \in \mathcal{H}_{e}^{1}$ (for $k \geq 1$ ) and $\bar{\phi}[k]=\oplus_{\sigma \geq 2 k+1} \bar{\phi}_{\sigma}[k] \in \mathcal{H}_{e}^{1}$ (for $k \geq 0$ ), such that:

$$
\begin{aligned}
s\left(\phi_{\sigma}[k]\right)=s\left(\bar{\phi}_{\sigma}[k]\right)=\sigma & , \quad g\left(\phi_{\sigma}[k]\right)=g\left(\bar{\phi}_{\sigma}[k]\right)=\sigma(\bmod 2) \\
g h\left(\phi_{\sigma}[k]\right)=\sigma-2 k & , \quad g h\left(\bar{\phi}_{\sigma}[k]\right)=2 k-\sigma
\end{aligned}
$$

for all $k \geq 0$, where $g h$ stands for the ghost number. We have $g h\left(\phi_{\sigma}[k]\right)+g h\left(\bar{\phi}_{\sigma}[k]\right)=0$ for all $k, \sigma$. Note that the ghost number $g h$ coincides with $s$ only for the original BV fields $\phi_{\sigma}[0]=\phi_{\sigma}$. The interpretation of the various fields is as follows. Remember that $\phi_{0}[0]=\phi_{0}$ is the physical field, while $\phi_{\sigma}[0]$ with $\sigma \geq 1$ are the ghosts. Then $\bar{\phi}_{\sigma}[0]$ (with $\sigma \geq 1$ ) are antighosts for these ghosts. The fields $\phi_{\sigma}[1](\sigma \geq 2)$ are first 
level extraghosts, i.e. ghosts for the antighosts $\bar{\phi}_{\sigma}[0]$ with $\sigma \geq 2$ (note that there is no extraghost associated with the first antighost $\left.\bar{\phi}_{1}[0]\right)$. Moreover, $\bar{\phi}_{\sigma}[1](\sigma \geq 3)$ are antighosts for those extraghosts $\phi_{\sigma}[1]$ which have $\sigma \geq 3$ (again the first extraghost $\phi_{2}[1]$ is left unpaired). At the second level, we introduce new extraghosts $\phi_{\sigma}[2]$ and their antighosts $\bar{\phi}_{\sigma}[2]$ and so on for higher levels. In general, $\bar{\phi}_{\sigma}[k]$ are antighosts for the $k^{t h}$ level extraghosts $\phi_{\sigma}[k]$ which have $\sigma \geq 2 k+1$. These fields can be arranged in a triangular field diagram [22], as shown in figure 2. This diagram will contain a finite number of nodes for a system based on a finite number of flat bundles $E_{n}$.

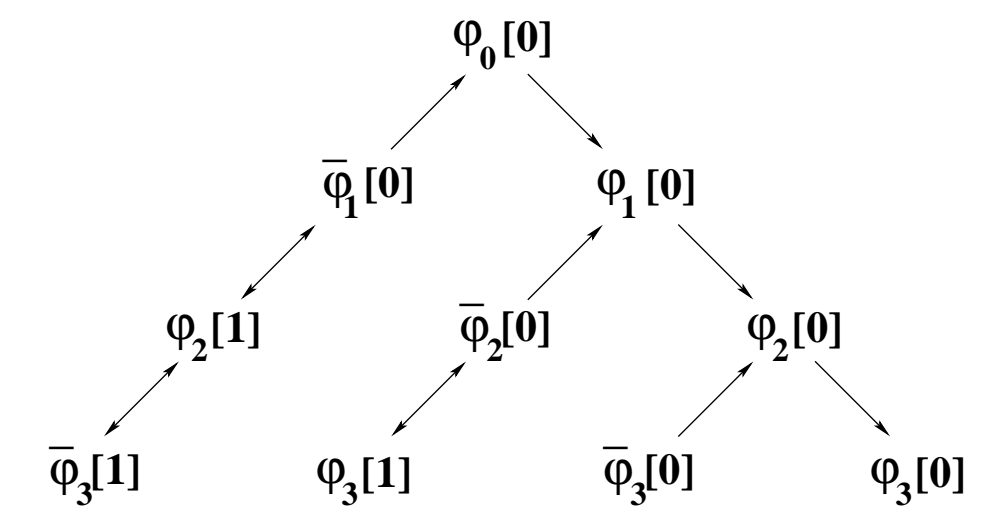

Figure 2: The triangular field diagram.

We also have the associated antifields $\phi^{*}[k]=\oplus_{\sigma \geq 2 k} \phi_{\sigma}^{*}[k] \in \mathcal{H}_{e}^{1}$ and $\bar{\phi}^{*}[k]=$ $\oplus_{\sigma \geq 2 k+1} \bar{\phi}_{\sigma}^{*}[k] \in \mathcal{H}_{e}^{1}($ for $k \geq 0)$, with:

$$
\begin{array}{cl}
s\left(\phi_{\sigma}^{*}[k]\right)=s\left(\bar{\phi}_{\sigma}^{*}[k]\right)=-\sigma-1 & , \quad g\left(\phi_{\sigma}^{*}[k]\right)=g\left(\bar{\phi}_{\sigma}^{*}[k]\right)=\sigma+1(\bmod 2) \\
g h\left(\phi_{\sigma}^{*}[k]\right)=2 k-\sigma-1 & , \quad g h\left(\bar{\phi}_{\sigma}^{*}[k]\right)=\sigma-2 k-1
\end{array}
$$

for all $k \geq 0$. Note that $\phi_{\sigma}^{*}[0]=\phi_{\sigma}^{*}$ are the original antifields. Finally, we introduce auxiliary fields $\pi[k]=\oplus_{\sigma \geq 2 k} \pi_{\sigma}[k] \in \mathcal{H}^{0}$ (for $k \geq 1$ ) and $\bar{\pi}[k]=\oplus_{\sigma \geq 2 k+1} \bar{\pi}_{\sigma}[k] \in \mathcal{H}^{0}$ (for $k \geq 0$ ) and their antifields $\pi^{*}[k]=\oplus_{\sigma \geq 2 k} \pi_{\sigma}^{*}[k] \in \mathcal{H}^{0}($ for $k \geq 1$ ) and $\bar{\pi}[k]=$ $\oplus_{\sigma \geq 2 k+1} \bar{\pi}_{\sigma}^{*}[k] \in \mathcal{H}^{0}$ (for $k \geq 0$ ), such that:

$$
\begin{array}{ll}
s\left(\pi_{\sigma}[k]\right)=s\left(\bar{\pi}_{\sigma}[k]\right)=\sigma & , \quad g\left(\pi_{\sigma}[k]\right)=g\left(\bar{\pi}_{\sigma}[k]\right)=\sigma+1(\bmod 2) \\
g h\left(\pi_{\sigma}[k]\right)=1+\sigma-2 k & , \quad g h\left(\bar{\pi}_{\sigma}[k]\right)=1-\sigma+2 k
\end{array}
$$

and:

$$
\begin{array}{cll}
s\left(\pi_{\sigma}^{*}[k]\right)=s\left(\bar{\pi}_{\sigma}^{*}[k]\right)=-1-\sigma & , \quad g\left(\pi_{\sigma}^{*}[k]\right)=g\left(\bar{\pi}_{\sigma}^{*}[k]\right)=\sigma(\bmod 2) \\
g h\left(\pi_{\sigma}^{*}[k]\right)=2 k-\sigma-2 & , \quad g h\left(\bar{\pi}_{\sigma}^{*}[k]\right)=\sigma-2 k-2 .
\end{array}
$$


The fields $\left(\pi_{\sigma}[k], \phi_{\sigma}[k]\right)$ (for $\left.k \geq 1\right)$ and $\left(\bar{\phi}_{\sigma}[k], \bar{\pi}_{\sigma}[k]\right)$ (for $k \geq 0$ ) form so-called trivial pairs [22].

It will convenient for what follows to decompose all fields into harmonic and nonharmonic components, according to the Hodge decomposition:

$$
\mathcal{H}_{e}=N_{e} \oplus K_{e} \quad \text { where } N_{e}=i m d_{e} \oplus i m d_{e}^{\dagger} .
$$

Thus, we write:

$$
\begin{array}{lll}
\phi_{\sigma}[k]=\phi_{\sigma}^{N}[k] \oplus \phi_{\sigma}^{K}[k] & , & \pi_{\sigma}[k]=\pi_{\sigma}^{N}[k] \oplus \pi_{\sigma}^{K}[k] \\
\phi_{\sigma}^{*}[k]=\phi_{\sigma}^{* N}[k] \oplus \phi_{\sigma}^{* K}[k] & , & \pi_{\sigma}^{*}[k]=\pi_{\sigma}^{* N}[k] \oplus \pi_{\sigma}^{* K}[k],
\end{array}
$$

with $\phi^{N}, \pi^{N}, \phi^{* N}, \pi^{* N} \in N_{e}$ and $\phi^{K}, \pi^{K}, \phi^{* K}, \pi^{* K} \in K_{e}$ (and similarly for the antighosts and their antifields and auxiliary fields). Note that $\phi^{N}$ decomposes further as $\phi^{U} \oplus \phi^{M}$, where $\phi^{U}$ and $\phi^{M}$ are the exact and coexact pieces; similar decompositions hold for all other fields.

Observation Due to rank constraints, many extraghost components in a component formalism (like the one used for a simple example in the appendix) will generally vanish. In this sense, the matrix of extraghosts is sparse; similar remarks apply for the auxiliary fields. (Because of this, the proper interpretation of various formulae below is to restrict them to the range of $\sigma$ for which the corresponding fields are non-vanishing. To simplify notation, we shall not indicate such ranges explicitly.) The position of nonvanishing components in the extraghost matrix generally corresponds to ghost degrees larger than 3 or smaller than zero (this is due to the shift in equation (2.3)). The reader can easily convince herself that a general system (for example, a system containing 3 bundle components $E_{n}$ of different grades) leads to a rather complicated distribution of non-vanishing entries and ghost numbers. The point of the formalism of the present section is that it automatically takes such complicated distributions into account, for an arbitrary system of bundles $E_{n}$ - thereby allowing for a general (or 'universal') formulation of the gauge-fixing procedure.

\subsection{A gauge-fermion of 'delta-function' type}

We next discuss a choice of gauge-fixing fermion which leads to the gauge used in [8]. Due to the presence of harmonic modes, our gauge-fixing procedure will be a bit involved, so we shall introduce it in two steps. The gauge-fixing fermion will be of deltafunction (or Landau) type. A more general family of gauges (of so-called weighted, or Feynman type) is briefly discussed in the appendix; it leads to the same results as the Landau-type fermion, after taking an appropriate limit. 


\subsubsection{Delta-function gauge-fixing in the absence of zero modes}

Let us first consider the case when the cohomology $H_{d}^{*}(\mathcal{H})$ is trivial in all degrees. In this situation, one has:

$$
\operatorname{kerd}_{e}=i m d_{e}, \operatorname{kerd}_{e}^{\dagger}=i m d_{e}^{\dagger}, \quad \mathcal{H}_{e}=N_{e}=i m d_{e} \oplus i m d_{e}^{\dagger}
$$

and the harmonic subspace $K_{e}$ vanishes. In particular, $\phi$ coincides with $\phi^{N}$, and similarly for all other fields.

Following standard gauge-fixing procedure, we consider the complete action $S_{c}=$ $S_{e}+S_{\text {aux }}$, where the auxiliary action is given by:

$$
S_{\text {aux }}=\operatorname{Re} \int_{L} \operatorname{str}_{e}\left(\sum_{k \geq 1} \sum_{\sigma \geq 2 k} \pi_{\sigma}^{N}[k] * \phi_{\sigma}^{* N}[k]+\sum_{k \geq 0} \sum_{\sigma \geq 2 k+1} \bar{\pi}_{\sigma}^{N}[k] * \bar{\phi}_{\sigma}^{* N}[k]\right) .
$$

It is easy to check that $S_{a u x}$ has ghost number zero and is Grassmann-even.

Consider the following gauge-fixing fermion:

$$
\Psi=\operatorname{Re} \int_{L} \operatorname{str}_{e} \sum_{k \geq 0}\left(\sum_{\sigma \geq 2 k} \bar{\phi}_{\sigma+1}^{N}[k] * d_{e} c_{e} \phi_{\sigma}^{N}[k]+\sum_{\sigma \geq 2 k+1} \phi_{\sigma+1}^{N}[k+1] * d_{e} c_{e} \bar{\phi}_{\sigma}^{N}[k]\right)
$$

It is easy to check that $\Psi$ is Grassmann-odd and satisfies $g h(\Psi)=-1$. The gauge-fixed action is obtained in two steps.

Elimination of antifields First, one must eliminate all antifields with the help of the gauge-fixing fermion:

$$
\phi_{\sigma}^{* N}[k]=\frac{\delta \Psi}{\delta \phi_{\sigma}^{N}[k]} \quad, \quad \bar{\phi}_{\sigma}^{* N}[k]=\frac{\delta \Psi}{\delta \bar{\phi}_{\sigma}^{N}[k]}
$$

as well as:

$$
\pi_{\sigma}^{* N}[k]=\bar{\pi}_{\sigma}^{* N}[k]=0
$$

The functional derivatives in the first equation are defined as in (3.30). Relations (4.10) lead to the equations ${ }^{2}$ :

$$
\begin{aligned}
& \phi_{\sigma}^{* N}[k]=c_{e} d_{e} \bar{\phi}_{\sigma+1}^{N}[k]-d_{e} c_{e} \bar{\phi}_{\sigma-1}^{N}[k-1] \\
& \bar{\phi}_{\sigma}^{* N}[k]=c_{e} d_{e} \phi_{\sigma+1}^{N}[k+1]-d_{e} c_{e} \phi_{\sigma-1}^{N}[k],
\end{aligned}
$$

\footnotetext{
${ }^{2}$ To arrive at these expressions, we used the identity:$$
\operatorname{Re}\left\langle\hat{u}, d_{e} c_{e} \hat{v}\right\rangle_{e}=(-1)^{(\operatorname{deg} \hat{u}+1) \operatorname{deg} \hat{v}} \operatorname{Re}\left\langle c_{e} d_{e} \hat{u}, \hat{v}\right\rangle_{e},
$$

which follows from (3.25). 
where we defined $\bar{\phi}_{\sigma}^{N}[-1]:=0$. Substituting (4.12) into $S_{c}$ gives the gauge-fixed action $S_{g f}=S_{e}\left(\phi_{\sigma}^{N}, \phi_{\sigma}^{* N}=\frac{\delta \Psi}{\delta \phi_{\sigma}^{N}}\right)+S_{a u x, g f}$, where:

$$
S_{a u x, g f}=S_{a u x}\left(\phi_{\sigma}^{* N}[k]=\frac{\delta \Psi}{\delta \phi_{\sigma}^{N}[k]}, \bar{\phi}_{\sigma}^{* N}[k]=\frac{\delta \Psi}{\delta \bar{\phi}_{\sigma}^{N}[k]}\right) .
$$

According to the general formalism, correlation functions can now be computed as:

$$
\left\langle\left\langle\mathcal{O}_{1} \ldots \mathcal{O}_{k}\right\rangle\right\rangle=\int \prod_{k, \sigma} \mathcal{D}\left[\phi_{\sigma}^{N}[k]\right] \mathcal{D}\left[\bar{\phi}_{\sigma}^{N}[k]\right] \mathcal{D}\left[\pi_{\sigma}^{N}[k]\right] \mathcal{D}\left[\bar{\pi}_{\sigma}^{N}[k]\right] e^{-i \lambda S_{g f}} \mathcal{O}_{1} \ldots \mathcal{O}_{k}
$$

Integration of auxiliary fields The next step is to notice that the equations of motion for the auxiliary fields $\pi_{\sigma}^{N}[k]$ and $\bar{\pi}_{\sigma}^{N}[k]$ impose the conditions:

$$
\begin{aligned}
& \phi_{\sigma}^{* N}[k+1]=\frac{\delta S_{a u x, g f}}{\delta \phi_{\sigma}^{N}[k+1]}=0 \Leftrightarrow d_{e} c_{e} \bar{\phi}_{\sigma-1}^{N}[k]=c_{e} d_{e} \bar{\phi}_{\sigma+1}^{N}[k+1] \quad(k \geq 0, \sigma \geq 2 k+2) \\
& \bar{\phi}_{\sigma}^{* N}[k]=\frac{\delta S_{a u x, g f}}{\delta \bar{\phi}_{\sigma}^{N}[k]}=0 \Leftrightarrow d_{e} c_{e} \phi_{\sigma-1}^{N}[k]=c_{e} d_{e} \phi_{\sigma+1}^{N}[k+1] \quad(k \geq 0 \quad, \quad \sigma \geq 2 k+1),
\end{aligned}
$$

which follow by integrating out $\pi_{\sigma}^{N}[k](k \geq 1)$ and $\bar{\pi}_{\sigma}^{N}[k](k \geq 0)$. Note that the auxiliary action does not contain terms in $\pi^{N}[0]$ (in fact, there are no such auxiliary fields), which is why we shifted $k$ by +1 in the first equation with respect to (4.12). In particular, the only condition imposed on $\bar{\phi}^{N}[0]$ is $d_{e} c_{e} \bar{\phi}_{\sigma}^{N}[0]=c_{e} d_{e} \bar{\phi}_{\sigma+2}^{N}[1]$. On the other hand, $S_{\text {aux }}$ does contain terms in $\bar{\pi}^{N}[0]$, which explains the range of $k$ used in the second equation.

At the path integral level, this can be understood as follows. Consider a correlator (4.14) of observables $\mathcal{O}_{j}$ which are independent of the auxiliary fields. Since the auxiliary fields appear linearly in the gauge-fixed action, one can perform the path integral over $\pi_{\sigma}^{N}[k]$ and $\bar{\pi}_{\sigma}^{N}[k]$. Due to the form of $S_{a u x, g f}$, this produces delta-function factors $\delta\left(\frac{\delta \Psi}{\delta \phi_{\sigma}[k]}\right)$ and $\delta\left(\frac{\delta \Psi}{\delta \bar{\phi}_{\sigma}[k]}\right)$, which restrict the path integral to fields satisfying the constraints (4.15). This effectively implements (4.15) at the level of all correlators, as long as we insist that observables should be independent of the auxiliary fields. Note that after integrating out $\pi$ and $\bar{\pi}$, the term $S_{a u x, g f}$ is completely eliminated from the gauge-fixed action. Thus:

$$
\begin{aligned}
\left\langle\left\langle\mathcal{O}_{1} \ldots \mathcal{O}_{k}\right\rangle\right\rangle & =\int \prod_{k, \sigma} \mathcal{D}\left[\phi_{\sigma}^{N}[k]\right] \mathcal{D}\left[\bar{\phi}_{\sigma}^{N}[k]\right] e^{-i \lambda S_{e, g f}} \mathcal{O}_{1} \ldots \mathcal{O}_{k} \\
& \prod_{k \geq 0} \prod_{\sigma \geq 2 k+1} \delta\left(d_{e} c_{e} \phi_{\sigma-1}^{N}[k]-c_{e} d_{e} \phi_{\sigma+1}^{N}[k+1]\right) \prod_{k \geq 0} \prod_{\sigma \geq 2 k+2} \delta\left(d_{e} c_{e} \bar{\phi}_{\sigma-1}^{N}[k]-c_{e} d_{e} \bar{\phi}_{\sigma+1}^{N}[k+1]\right) .
\end{aligned}
$$

To eliminate the delta-functions, we decompose $\phi^{N}$ and $\bar{\phi}^{N}$ into their exact and coexact components (denoted by superscripts $U$ and $M$, respectively). Since $d_{e} c_{e} / c_{e} d_{e}$ vanishes on coexact/exact elements, and restricts to a bijection on the exact/coexact subspaces 
of $\mathcal{H}_{e}$, we can separate the delta-functions as follows:

$$
\begin{gathered}
\delta\left(d_{e} c_{e} \phi_{\sigma-1}^{N}[k]-c_{e} d_{e} \phi_{\sigma+1}^{N}[k+1]\right)=\delta\left(d_{e} c_{e} \phi_{\sigma-1}^{U}[k]\right) \delta\left(c_{e} d_{e} \phi_{\sigma+1}^{M}[k+1]\right)= \\
=\left[\operatorname{det}_{\mathbf{R}, s=\sigma-1}^{\prime}\left(d^{\dagger}\right) \operatorname{det}_{\mathbf{R}, s=\sigma+1}^{\prime}\left(d^{\dagger} d\right)\right]^{\frac{(-1)^{\sigma}}{2}} \delta\left(\phi_{\sigma-1}^{U}[k]\right) \delta\left(\phi_{\sigma+1}^{M}[k+1]\right) \\
\delta\left(d_{e} c_{e} \bar{\phi}_{\sigma-1}^{N}[k]-c_{e} d_{e} \bar{\phi}_{\sigma+1}^{N}[k+1]\right)=\delta\left(d_{e} c_{e} \bar{\phi}_{\sigma-1}^{U}[k]\right) \delta\left(c_{e} d_{e} \bar{\phi}_{\sigma+1}^{M}[k+1]\right)= \\
=\left[\operatorname{det}_{\mathbf{R}, s=\sigma-1}^{\prime}\left(d d^{\dagger}\right) \operatorname{det}_{\mathbf{R}, s=\sigma+1}^{\prime}\left(d^{\dagger} d\right)\right]^{\frac{(-1)^{\sigma}}{2}} \delta\left(\bar{\phi}_{\sigma-1}^{U}[k]\right) \delta\left(\bar{\phi}_{\sigma+1}^{M}[k+1]\right) .
\end{gathered}
$$

To derive the last equalities, we noticed that $\delta\left(d_{e} c_{e} u_{\sigma}^{U}\right)=\operatorname{det}_{\mathbf{R}}^{\prime}\left(\left[(d c)_{s=\sigma}\right]^{t}(d c)_{s=\sigma}\right)^{\frac{(-1)^{\sigma+1}}{2}} \delta\left(u_{\sigma}^{U}\right)$ and $\delta\left(c_{e} d_{e} u_{\sigma}^{M}\right)=\operatorname{det}_{\mathbf{R}}^{\prime}\left(\left[(c d)_{s=\sigma}\right]^{t}(c d)_{s=\sigma}\right)^{\frac{(-1)^{\sigma+1}}{2}} \delta\left(u_{\sigma}^{M}\right)$, for $u^{U} \in \operatorname{imd}_{e}(s=\sigma) \cap \mathcal{H}_{e}^{1}$ and $u^{M} \in i m d_{e}^{\dagger}(s=\sigma) \cap \mathcal{H}_{e}^{1}$, where the prime denotes restriction to the orthocomplement of the relevant operator and ${ }^{t}$ denotes the transpose of a real-linear operator with respect to the induced Euclidean scalar product $(.,)=.R e<., .>$ on $\mathcal{H}$. The determinant factors follow from the transformation rule of the associated measure, which is controlled by the volume forms constructed with the metric $(.$,$) ; the exponents are controlled by$ the Grassmannality of $u_{\sigma}$ (which is equal to $\left.\sigma(\bmod 2)\right)$. To simplify these factors, we used the relations:

$$
(d c u, v)=(-1)^{1+s(u)}(u, c d v) \quad, \quad(c d u, v)=(-1)^{s(u)}(u, c d v),
$$

which can be checked by using the relation between the Hermitian product $h$ and the bilinear form $<.,$.$\rangle , as well as the properties of the later. These relations imply:$

$$
\left[(d c)_{s=\sigma}\right]^{t}=(-1)^{1+\sigma}(d c)_{s=-2-\sigma} \quad, \quad\left[(c d)_{s=\sigma}\right]^{t}=(-1)^{\sigma}(c d)_{s=-\sigma}
$$

which in turn give:

$$
\left[(d c)_{s=\sigma}\right]^{t}(d c)_{s=\sigma}=d d^{\dagger} \quad, \quad\left[(c d)_{s=\sigma}\right]^{t}(c d)_{s=\sigma}=d^{\dagger} d
$$

We note that $(d c)_{s=\sigma}$ is a map $\mathcal{H}(\sigma) \rightarrow \mathcal{H}(-2-\sigma)$ and $(c d)_{s=\sigma}$ is a map $\mathcal{H}(\sigma) \rightarrow \mathcal{H}(-\sigma)$, while their adjoints $\left[(d c)_{s=\sigma}\right]^{t}$ and $\left[(c d)_{s=\sigma}\right]^{t}$ give reversed arrows between the same subspaces.

Combining everything in equation (4.17), we obtain a factor $P Q$, where:

$$
\begin{aligned}
P & :=\prod_{k \geq 0} \prod_{\sigma \geq 2 k+1} \delta\left(\phi_{\sigma-1}^{U}[k]\right) \delta\left(\phi_{\sigma+1}^{M}[k+1]\right) \times \prod_{k \geq 0} \prod_{\sigma \geq 2 k+2} \delta\left(\bar{\phi}_{\sigma-1}^{U}[k]\right) \delta\left(\bar{\phi}_{\sigma+1}^{M}[k+1]\right)= \\
& =\prod_{\sigma \geq 0} \delta\left(\phi_{\sigma}^{U}[0]\right) \prod_{\sigma \geq 1} \delta\left(\bar{\phi}_{\sigma}^{U}[0]\right) \times \prod_{k \geq 1} \prod_{\sigma \geq 2 k} \delta\left(\phi_{\sigma}^{N}[k]\right) \prod_{k \geq 1} \prod_{\sigma \geq 2 k+1} \delta\left(\bar{\phi}_{\sigma}^{N}[k]\right),
\end{aligned}
$$

while:

$$
\begin{aligned}
Q & :=\prod_{k \geq 0} \prod_{\sigma \geq 2 k+1}\left[\operatorname{det}_{\mathbf{R}, s=\sigma-1}^{\prime}\left(d d^{\dagger}\right) \operatorname{det}_{\mathbf{R}, s=\sigma+1}^{\prime}\left(d^{\dagger} d\right)\right]^{\frac{(-1)^{\sigma}}{2}} \prod_{k \geq 0} \prod_{\sigma \geq 2 k+2}\left[\operatorname{det}_{\mathbf{R}, s=\sigma-1}^{\prime}\left(d d^{\dagger}\right) \operatorname{det}_{\mathbf{R}, s=\sigma+1}^{\prime}\left(d^{\dagger} d\right)\right]^{\frac{(-1)^{\sigma}}{2}} \\
& =\prod_{k \geq 0} \prod_{\sigma \geq 2 k+1}\left[\operatorname{det}_{s=\sigma-1}^{\prime}\left(d d^{\dagger}\right) \operatorname{det}_{s=\sigma+1}^{\prime}\left(d^{\dagger} d\right)\right]^{(-1)^{\sigma}} \prod_{k \geq 0} \prod_{\sigma \geq 2 k+2}\left[\operatorname{det}_{s=\sigma-1}^{\prime}\left(d d^{\dagger}\right) \operatorname{det}_{s=\sigma+1}^{\prime}\left(d^{\dagger} d\right)\right]^{(-1)^{\sigma}},(4.22)
\end{aligned}
$$


where in the second line we passed to complex determinants. To simplify $Q$, we use the fact that the operators $\left.d d^{\dagger}\right|_{k e r\left(d^{\dagger}\right)^{\perp}(s=\sigma)}$ and $\left.d^{\dagger} d\right|_{k e r(d)^{\perp}(s=\sigma+1)}$ are isospectral (this is proved in [9]), which implies that their determinants are equal. Thus:

$$
Q=\prod_{k \geq 0} \prod_{\sigma \geq 2 k+1}\left[\operatorname{det}_{s=\sigma}^{\prime}\left(d^{\dagger} d\right) d e t_{s=\sigma+1}^{\prime}\left(d^{\dagger} d\right)\right]^{(-1)^{\sigma}} \prod_{k \geq 0} \prod_{\sigma \geq 2 k+2}\left[\operatorname{det}_{s=\sigma}^{\prime}\left(d^{\dagger} d\right) \operatorname{det}_{s=\sigma+1}^{\prime}\left(d^{\dagger} d\right)\right]^{(-1)^{\sigma}}=\frac{1}{I}
$$

where:

$$
I:=\prod_{\sigma>0} d e t_{s=\sigma}^{\prime}\left(d^{\dagger} d\right)^{(-1)^{\sigma+1}}
$$

The gauge-fixed path integral Returning to expression (4.16), the factor (4.21) eliminates all integrals over $\phi_{\sigma}^{N}[k]$ and $\bar{\phi}_{\sigma}^{N}[k]$ (with $k \geq 1$ ) by setting these fields to zero, and restricts the remaining integrals to integrals over the coexact components $\phi^{M}[0]=\phi^{M}$ and $\bar{\phi}^{M}[0]:=\bar{\phi}^{M}$. On the other hand, $Q$ produces the prefactor $\frac{1}{I}$. Since the contribution $S_{a u x}$ was eliminated when integrating out the auxiliary fields, the only other effect of gauge-fixing is to impose the condition $\phi_{\sigma}^{*}=c_{e} d_{e} \bar{\phi}_{\sigma+1}^{M}$ in the extended action $S_{e}$. Remembering that $\phi_{\sigma}^{*}=\hat{\phi}_{-1-\sigma}$, this also reads:

$$
\hat{\phi}_{-\sigma}=c_{e} d_{e} \bar{\phi}_{\sigma}^{M} \quad \text { for all } \quad \sigma>0
$$

Recalling the notations $\hat{\phi}_{+}:=\oplus_{\sigma \geq 0} \phi_{\sigma}, \hat{\phi}_{-}:=\oplus_{\sigma<0} \phi_{\sigma}$ and $\bar{\phi}:=\oplus_{\sigma>0} \bar{\phi}_{\sigma}$, we have $\hat{\phi}_{-}=c_{e} d_{e} \bar{\phi}$. Note that $c_{e} d_{e}$ gives a bijection between $i m d_{e}^{\dagger}(s>0)$ and $i m d_{e}^{\dagger}(s<0)$, so the last relation can be viewed as a (differential) linear change of variables.

Thus the gauge-fixed action reduces to:

$$
S_{g f}=S_{e}\left(\phi_{\sigma}^{M}, \phi_{\sigma}^{* M}=c_{e} d_{e} \bar{\phi}_{\sigma+1}^{M}\right)=S_{e}\left(\hat{\phi}_{+}^{M}, \hat{\phi}_{-}^{M}=c_{e} d_{e} \bar{\phi}^{M}\right)
$$

where $\hat{\phi}_{+}^{M} \in i m d_{e}^{\dagger}(s \geq 0)$ and $\bar{\phi}^{M} \in i m d_{e}^{\dagger}(s>0)$. The gauge-fixed, extended field $\hat{\phi}^{M}=\hat{\phi}_{+}^{M} \oplus \hat{\phi}_{-}^{M}=\hat{\phi}_{+}^{M} \oplus\left(c_{e} d_{e} \bar{\phi}^{M}\right)$ has the expansion:

$$
\hat{\phi}^{M}=\ldots+c_{e} d_{e} \bar{\phi}_{2}^{M}+c_{e} d_{e} \bar{\phi}_{1}^{M}+\phi_{0}^{M}+\phi_{1}^{M}+\phi_{2}^{M}+\ldots
$$

Thus the sole effect of gauge-fixing is to replace the extended field $\hat{\phi}=\hat{\phi}_{+} \oplus \hat{\phi}_{-} \in \mathcal{H}_{e}$ with the field $\hat{\phi}_{+}^{M} \oplus\left(c_{e} d_{e} \bar{\phi}^{M}\right) \in i m d_{e}^{\dagger}$. This is very similar to what happens for acyclic backgrounds in the ungraded case [21].

Conclusion In the acyclic case, correlators have the form:

$$
\left\langle\left\langle\mathcal{O}_{1} \ldots \mathcal{O}_{k}\right\rangle\right\rangle=\frac{1}{I} \int \prod_{\sigma \geq 0} \mathcal{D}\left[\phi_{\sigma}^{M}\right] \prod_{\sigma \geq 1} \mathcal{D}\left[\bar{\phi}_{\sigma}^{M}\right] e^{-i \lambda S_{e}\left(\phi_{\sigma}^{*}=c_{e} d_{e} \bar{\phi}_{\sigma+1}^{M}, \phi_{\sigma}=\phi_{\sigma}^{M}\right)} \mathcal{O}_{1} \ldots \mathcal{O}_{k}
$$

where $I$ is given in equation (4.24). 


\subsubsection{Extension to the case with zero modes}

According to Hodge theory, the field space now splits into

$$
\mathcal{H}_{e}=N_{e} \oplus K_{e}
$$

where $N_{e}=i m d_{e} \oplus i m d_{e}^{\dagger}$ is, as before, the collection of all exact and coexact field configurations while $K_{e}$ is the collection of all harmonic modes. Accordingly, we write $\hat{\phi}=\hat{\phi}^{N} \oplus \hat{\phi}^{K}$ (with $\hat{\phi}^{N} \in N_{e}$ and $\hat{\phi}^{K} \in K_{e}$ ) and perform gauge fixing for each component separately, by adding trivial pairs along each subspace. The full auxiliary action reads:

$$
\begin{aligned}
S_{\text {aux }} & =\operatorname{Re} \int_{L} s \operatorname{st}_{e}\left(\sum_{k \geq 1} \sum_{\sigma \geq 2 k} \pi_{\sigma}^{N}[k] * \phi_{\sigma}^{* N}[k]+\sum_{k \geq 0} \sum_{\sigma \geq 2 k+1} \bar{\pi}_{\sigma}^{N}[k] * \bar{\phi}_{\sigma}^{* N}[k]\right) \\
& +\operatorname{Re} \int_{L} \operatorname{str}\left(\sum_{k \geq 1} \sum_{\sigma \geq 2 k} \pi_{\sigma}^{K}[k] * A_{-1-\sigma}[k]^{1 / 2} \phi_{\sigma}^{* K}[k]+\sum_{k \geq 0} \sum_{\sigma \geq 2 k+1} \bar{\pi}_{\sigma}^{K}[k] * A_{-1-\sigma}[k]^{1 / 2} \bar{\phi}_{\sigma}^{* K}[k]\right) \\
& +\operatorname{Re} \int_{L} s \operatorname{st} \sum_{k \geq 0}\left(\sum_{\sigma \geq 2 k} \lambda_{\sigma}^{K}[k] * A_{-1-\sigma}[k]^{1 / 2} \bar{\mu}_{\sigma}^{* K}[k]+\sum_{\sigma \geq 2 k+1} \bar{\lambda}_{\sigma}^{K}[k] * A_{-1-\sigma}[k]^{1 / 2} \mu_{\sigma}^{* K}[k]\right)
\end{aligned}
$$

where $A_{\sigma}[k]$ are some (strictly) positive operators on $K(\sigma)$, while the new fields $\lambda^{K}$, $\mu^{K}, \bar{\lambda}^{K}$ and $\bar{\mu}^{K}$ have charges:

$$
\begin{aligned}
s\left(\lambda_{\sigma}^{K}[k]\right)=s\left(\bar{\lambda}_{\sigma}^{K}[k]\right)=\sigma & , s\left(\mu_{\sigma}^{K}[k]\right)=s\left(\bar{\mu}_{\sigma}^{K}[k]\right)=\sigma \\
g\left(\lambda_{\sigma}^{K}[k]\right)=g\left(\bar{\lambda}_{\sigma}^{K}[k]\right)=\sigma(\bmod 2) & , g\left(\mu_{\sigma}^{K}[k]\right)=g\left(\bar{\mu}_{\sigma}^{K}[k]\right)=\sigma+1(\bmod 2) \\
g h\left(\lambda_{\sigma}^{K}[k]\right)=2 k-\sigma & , g h\left(\mu_{\sigma}^{K}[k]\right)=\sigma-2 k-1 \\
g h\left(\lambda_{\sigma}^{K}[k]\right)=\sigma-2 k & , g h\left(\bar{\mu}_{\sigma}^{K}[k]\right)=2 k-\sigma-1 .
\end{aligned}
$$

Note that $\phi_{\sigma}^{K *}$ etc have $s=-1-\sigma$. As in [9], we shall choose $A_{\sigma}[k]$ such that:

$$
c A_{\sigma}[k]=A_{-1-\sigma}[k] c .
$$

This is possible because $c$ gives bijections between $K(\sigma)$ and $K(-1-\sigma)$.

The charge assignments in (4.31) follow from elementary considerations. For example, the BRST variation of $\bar{\mu}_{\sigma}^{K}[k]$ is $\lambda_{\sigma}^{K}[k]$, which implies that their Grassmann parities should be opposite, their $U(1)$ degrees should be the same (and consequently their $s$ numbers) while the ghost number of $\lambda_{\sigma}^{K}[k]$ should be larger by one unit than the ghost number of $\bar{\mu}_{\sigma}^{K}[k]$. Similar arguments hold for $\mu_{\sigma}^{K}[k]$ and $\bar{\lambda}_{\sigma}^{K}[k]$. The terms involving $\lambda$ and $\bar{\lambda}$ will allow us to gauge-fix the harmonic components of all fields $\phi$ and $\bar{\phi}$. The range of summation of $\sigma$ and $k$ implies that $\lambda$ will fix the harmonic components of $\phi$ while $\bar{\lambda}$ will fix the harmonic components of $\bar{\phi}$. Thus, the quantum numbers of $\lambda$ and $\bar{\lambda}$ are uniquely fixed which in turn fixes the rest. 
The simplest gauge is obtained by choosing a delta-type gauge fixing fermion for both harmonic and non-harmonic components. This has the form:

$$
\begin{gathered}
\Psi=\operatorname{Re} \int_{L} s t r_{e} \sum_{k \geq 0}\left(\sum_{\sigma \geq 2 k} \bar{\phi}_{\sigma+1}^{N}[k] * d_{e} c_{e} \phi_{\sigma}^{N}[k]+\sum_{\sigma \geq 2 k+1} \phi_{\sigma+1}^{N}[k+1] * d_{e} c_{e} \bar{\phi}_{\sigma}^{N}[k]\right) \\
+\operatorname{Re} \int_{L} s \operatorname{st}_{e} \sum_{k \geq 0}\left(\sum_{\sigma \geq 2 k}\left(c_{e} \bar{\mu}_{\sigma}^{K}[k]\right) * A_{\sigma}[k]^{1 / 2}\left(\phi_{\sigma}^{K}[k]-\phi_{\sigma}^{H}[k]\right)\right. \\
\left.+\sum_{\sigma \geq 2 k+1}\left(c_{e} \mu_{\sigma}^{K}[k]\right) * A_{\sigma}[k]^{1 / 2}\left(\bar{\phi}_{\sigma}^{K}[k]-\bar{\phi}_{\sigma}^{H}[k]\right)\right),
\end{gathered}
$$

where $\phi_{\sigma}^{H}[k]$ and $\bar{\phi}_{\sigma}^{H}[k]$ are some constant elements belonging to the appropriate harmonic subspaces (these constant shifts serve as parameters). In principle, for the terms in the first line above one could forget the superscript $N$ and consider the full field, because harmonic components are annihilated by $d_{e}$ and $d_{e} c_{e}$. These terms would then be invariant under shifting $\phi$ and $\bar{\phi}$ by harmonic forms. This residual gauge symmetry is then fixed by the terms in the second line. If one did not fix this extra invariance then the antifields would not be uniquely determined in terms of fields and the resulting kinetic term would still be degenerate.

The harmonic components of antifields are eliminated through the equations:

$$
\begin{aligned}
& \phi_{\sigma}^{K *}[k]=\frac{\delta \Psi}{\delta \phi_{\sigma}^{K}[k]}=c_{e} A_{\sigma}[k]^{1 / 2} \bar{\mu}_{\sigma}^{K}[k] \quad, \quad \bar{\phi}_{\sigma}^{K *}[k]=\frac{\delta \Psi}{\delta \bar{\phi}_{\sigma}^{K}[k]}=c_{e} A_{\sigma}[k]^{1 / 2} \mu_{\sigma}^{K}[k] \\
& \mu_{\sigma}^{K *}[k]=\frac{\delta \Psi}{\delta \mu_{\sigma}^{K}[k]}=c_{e} A_{\sigma}[k]^{1 / 2}\left(\bar{\phi}_{\sigma}^{K}[k]-\bar{\phi}_{\sigma}^{H}[k]\right), \quad \bar{\mu}_{\sigma}^{K *}[k]=\frac{\delta \Psi}{\delta \bar{\mu}_{\sigma}^{K}[k]}=c_{e} A_{\sigma}[k]^{1 / 2}\left(\phi_{\sigma}^{K}[k]-\phi_{\sigma}^{H}[k]\right),
\end{aligned}
$$

while the non-harmonic components are eliminated through (4.12).

Substituting these into the auxiliary action gives:

$$
\begin{aligned}
S_{a u x, g f}=\operatorname{Re} \int_{L} s t r_{e}( & \sum_{k \geq 0} \sum_{\sigma \geq 2 k} \lambda_{\sigma}^{K}[k] * c_{e} A_{\sigma}[k]\left(\phi_{\sigma}^{K}[k]-\phi_{\sigma}^{H}[k]\right) \\
& \left.+\sum_{k \geq 0} \sum_{\sigma \geq 2 k+1} \bar{\lambda}_{\sigma}^{K}[k] * c_{e} A_{\sigma}[k]\left(\bar{\phi}_{\sigma}^{K}[k]-\bar{\phi}_{\sigma}^{H}[k]\right)\right) \\
+ & \operatorname{Re} \int_{L} \operatorname{str}\left(\sum_{k \geq 1} \sum_{\sigma \geq 2 k} \pi_{\sigma}^{K}[k] * c_{e} A_{\sigma}[k] \bar{\mu}_{\sigma}^{K}[k]\right. \\
& \left.+\sum_{k \geq 0} \sum_{\sigma \geq 2 k+1} \bar{\pi}_{\sigma}^{K}[k] * c_{e} A_{\sigma}[k] \mu_{\sigma}^{K}[k]\right) \\
+ & S_{\text {aux }, g f}\left(\phi^{N}, \bar{\phi}^{N}\right),
\end{aligned}
$$

where the last term has the form given in $(4.13,4.8)$ and where we used relation $(4.32)$. 
Considering a correlator of operators independent of auxiliary fields, the path integral over $\lambda^{K}$ and $\bar{\lambda}^{K}$ produces:

$$
\begin{aligned}
& \prod_{k \geq 0 ; \sigma \geq 2 k} \delta\left(\phi_{\sigma}^{K}[k]-\phi_{\sigma}^{H}[k]\right) \prod_{k \geq 0 ; \sigma \geq 2 k+1} \delta\left(\bar{\phi}_{\sigma}^{K}[k]-\bar{\phi}_{\sigma}^{H}[k]\right) \times \\
& \times \prod_{k \geq 0 ; \sigma \geq 2 k} \operatorname{det}_{\mathbf{R}} A_{\sigma}[k]^{(-1)^{\sigma+1}} \prod_{k \geq 0 ; \sigma \geq 2 k+1} \operatorname{det}_{\mathbf{R}} A_{\sigma}[k]^{(-1)^{\sigma+1}},
\end{aligned}
$$

where we used the fact that the Grassmannality of $\phi_{\sigma}^{K}[k]$ and $\bar{\phi}_{\sigma}^{K}[k]$ equals $\sigma(\bmod 2)$.

On the other hand, the integral over $\pi^{K}, \mu^{K}$ and their barred counterparts gives:

$$
\prod_{k \geq 1 ; \sigma \geq 2 k} \operatorname{det}_{\mathbf{R}} A_{\sigma}[k]^{(-1)^{\sigma}} \prod_{k \geq 0 ; \sigma \geq 2 k+1} \operatorname{det}_{\mathbf{R}} A_{\sigma}[k]^{(-1)^{\sigma}}
$$

To arrive at this equation, we first performed the integral over $\mu^{K}$ and $\bar{\mu}^{K}$, which gives delta-function factors of the form $\delta\left(A_{\sigma}[k] \pi_{\sigma}^{K}[k]\right)$ and $\delta\left(A_{\sigma}[k] \pi_{\sigma}^{K}[k]\right)$, and we noticed that:

$$
\begin{aligned}
& \delta\left(A_{\sigma}[k] \pi_{\sigma}^{K}[k]\right)=\operatorname{det}_{\mathbf{R}} A_{\sigma}[k]^{(-1)^{\sigma}} \delta\left(\pi_{\sigma}^{K}[k]\right) \\
& \delta\left(A_{\sigma}[k] \pi_{\sigma}^{K}[k]\right)=\operatorname{det}_{\mathbf{R}} A_{\sigma}[k]^{(-1)^{\sigma}} \delta\left(\bar{\pi}_{\sigma}^{K}[k]\right),
\end{aligned}
$$

where we used the fact that the Grassmannality of $\pi_{\sigma}^{K}[k]$ and $\bar{\pi}_{\sigma}^{K}[k]$ is $\sigma+1(\bmod 2)$. The remaining integral over these fields eliminates the delta-function factors and gives the contribution (4.37).

Therefore, the final result of the integral over the harmonic sector of auxiliary fields is to produce a factor:

$$
\prod_{k \geq 0 ; \sigma \geq 2 k} \delta\left(\phi_{\sigma}^{K}[k]-\phi_{\sigma}^{H}[k]\right) \prod_{k \geq 0 ; \sigma \geq 2 k+1} \delta\left(\bar{\phi}_{\sigma}^{K}[k]-\bar{\phi}_{\sigma}^{H}[k]\right) \prod_{\sigma \geq 0}\left[\operatorname{det}_{\mathbf{R}} A_{\sigma}\right]^{(-1)^{\sigma+1}},
$$

while eliminating all but the last term in eq. (4.35). In the last relation, we defined $A_{\sigma}:=A_{\sigma}[0]$. The contribution (4.39) has the effect of killing all integrals over all harmonic components, while inducing a prefactor:

$$
J:=\prod_{\sigma \geq 0} \operatorname{det}_{\mathbf{R}} A_{\sigma}^{(-1)^{\sigma+1}}
$$

in the gauge-fixed path integral. For $k=0,(4.39)$ gives the gauge-fixing condition for massless modes:

$$
\phi_{\sigma}^{K}=\phi_{\sigma}^{H}, \bar{\phi}_{\sigma}^{K}=\bar{\phi}_{\sigma}^{H}
$$

where we defined $\phi_{\sigma}^{H}:=\phi_{\sigma}^{H}[0]$ and $\bar{\phi}_{\sigma}^{H}:=\bar{\phi}_{\sigma}^{H}[0]$. Since $\phi_{\sigma}^{K}$ are frozen to the values $\phi_{\sigma}^{H}$, we are left with the extended action $S_{e}\left(\phi_{\sigma}^{*}, \phi_{\sigma}=\phi_{\sigma}^{N} \oplus \phi_{\sigma}^{H}\right)$ and with the last term of the auxiliary action (4.35), which depends only on non-harmonic modes. Integrating out 
the non-harmonic auxiliary fields $\pi^{K}$ and $\bar{\pi}^{K}$ now has the effect discussed in the previous subsection, thereby eliminating the last term in the auxiliary action and producing the prefactor $\frac{1}{I}$ with $I$ given in equation (4.24). It also kills all integrals over extraghosts and their antighosts, restricts the integrals over ghosts and antighosts to their massive components and implements the condition $\phi_{\sigma}^{*}=c_{e} d_{e} \bar{\phi}_{\sigma+1}^{M}$ in the tree-level BV action $S_{e}$. The result is a gauge-fixed action given by $S_{e}\left(\phi_{\sigma}^{*}=c_{e} d_{e} \bar{\phi}_{\sigma}^{M}, \phi_{\sigma}=\phi_{\sigma}^{M} \oplus \phi_{\sigma}^{H}\right)$, and a prefactor equal to $J / I$ in front of the gauge-fixed path integral.

\subsection{Summary}

Combining everything, we find that the result of gauge fixing is as follows:

(1)The gauge-fixed action is given by:

$$
S_{g f}=S_{e}\left(\phi_{\sigma}^{*}=c_{e} d_{e} \bar{\phi}_{\sigma+1}^{M}, \phi_{\sigma}=\phi_{\sigma}^{M} \oplus \phi_{\sigma}^{H}\right)
$$

where the physical field and ghosts $\phi_{\sigma}^{M}(\sigma \geq 0)$ and the antighosts $\bar{\phi}_{\sigma}^{M}(\sigma>0)$ belong to $i m d_{e}^{\dagger}$, while $\phi_{\sigma}^{H}(\sigma \geq 0)$ are some fixed harmonic elements which play the role of parameters. The components $\phi_{\sigma}^{H}$ can be assembled into the element:

$$
\hat{\phi}_{+}^{H}=\oplus_{\sigma \geq 0} \phi_{\sigma}^{H} \in K_{e}(s \geq 0)
$$

The harmonic components of the antighosts are fixed to some irrelevant values, and do not enter the gauge-fixed action.

We also write:

$$
S_{g f}=S_{e}\left(\hat{\phi}_{-}=c_{e} d_{e} \bar{\phi}^{M}, \hat{\phi}_{+}=\hat{\phi}_{+}^{M} \oplus \hat{\phi}_{+}^{H}\right)
$$

where $\bar{\phi}^{M}=\sum_{\sigma>0} \bar{\phi}_{\sigma}^{M} \in i m d_{e}^{\dagger}(s>0)$. Note that $\hat{\phi}_{+}=\sum_{\sigma \geq 0} \hat{\phi}_{\sigma} \in i m d_{e}^{\dagger}(s \geq 0) \oplus K_{e}(\sigma \geq$ $0)$ and $\hat{\phi}_{-}=\sum_{\sigma<0} \hat{\phi}_{\sigma} \in i m d_{e}^{\dagger}(s<0)$.

(2) A prefactor of $J / I$, where:

$$
J=\prod_{\sigma \geq 0} \operatorname{det}_{\mathbf{R}} A_{\sigma}^{(-1)^{\sigma+1}} \text { and } I=\prod_{\sigma>0} \operatorname{det}_{s=\sigma}^{\prime}\left(d^{\dagger} d\right)^{(-1)^{\sigma+1}}
$$

is induced in the path integral measure. The data $A_{\sigma}:=A_{\sigma}[0]$ are (strictly) positive operators on the harmonic subspaces $K(\sigma)$, which play the role of gauge-fixing parameters for the zero modes. 


\subsubsection{Cohomological formalism for $A_{\sigma}$}

To make contact with the formalism of [9], we now express the data $A_{\sigma}$ in terms of equivalent data used in that paper. Let us fix $\sigma$ and consider the Hermitian metric $h_{\sigma}$ induced by $h$ on $K_{\sigma}$. It is easy to see that specifying a positive operator $A_{\sigma}$ on $K_{\sigma}$ is equivalent to specifying another Hermitian metric $g_{\sigma}$ on $K_{\sigma}$. Indeed, given such an operator one constructs $g_{\sigma}$ through:

$$
g_{\sigma}(u, v):=h_{\sigma}\left(\left(A_{\sigma}\right)^{2} u, v\right)=h_{\sigma}\left(A_{\sigma} u, A_{\sigma} v\right)
$$

Conversely, any Hermitian metric can be written in this form. To see this, consider bases $e_{i}$ and $e_{i}^{\prime}$ of $K_{\sigma}$ which are orthonormal with respect to $h_{\sigma}$, respectively $g_{\sigma}$. If $B_{\sigma}$ is the invertible operator which takes $e_{i}^{\prime}$ into $e_{i}$, then we have:

$$
h_{\sigma}\left(B_{\sigma}\left(e_{i}^{\prime}\right), B_{\sigma}\left(e_{j}^{\prime}\right)\right)=h_{\sigma}\left(e_{i}, e_{j}\right)=g_{\sigma}\left(e_{i}^{\prime}, e_{j}^{\prime}\right)=\delta_{i j} \Rightarrow g_{\sigma}(u, v)=h_{\sigma}\left(B_{\sigma} u, B_{\sigma} v\right) .
$$

Defining the positive operator $A_{\sigma}$ through $A_{\sigma}:=\left(B_{\sigma}^{\dagger} B_{\sigma}\right)^{1 / 2}$, we have $B_{\sigma}^{\dagger} B_{\sigma}=\left(A_{\sigma}\right)^{2}$ and $g_{\sigma}(u, v)=h_{\sigma}\left(B_{\sigma} u, B_{\sigma} v\right)=h_{\sigma}\left(\left(A_{\sigma}\right)^{2} u, v\right)$.

We next consider the Hodge isomorphism $f_{\sigma}: K_{\sigma} \rightarrow H_{\sigma}(\mathcal{H})=H_{d}^{1-\sigma}(\mathcal{H})$, given by associating to every harmonic element $u$ its cohomology class. It is clear that specifying a Hermitian metric $g_{\sigma}$ on $K_{\sigma}$ is equivalent to giving a Hermitian metric $h_{\sigma}^{H}$ on $H_{\sigma}(\mathcal{H})$, the two being related by:

$$
h_{\sigma}^{H}\left(f_{\sigma}(u), f_{\sigma}(v)\right)=g_{\sigma}(u, v)
$$

Combining these two observations, we see that the data $A_{\sigma}$ is equivalent to the specification of a metric $h_{\sigma}^{H}$ on $H_{\sigma}(\mathcal{H})$, the relation between these equivalent objects being given by:

$$
h_{\sigma}^{H}\left(f_{\sigma} u, f_{\sigma} v\right)=h_{\sigma}\left(\left(A_{\sigma}\right)^{2} u, v\right) .
$$

If $f_{\sigma}^{\dagger}: H_{\sigma}(\mathcal{H}) \rightarrow K_{\sigma}$ is the Hermitian conjugate of $f_{\sigma}$ with respect to the metrics $h_{\sigma}$ and $h_{\sigma}^{H}$, then $h_{\sigma}^{H}\left(f_{\sigma} u, f_{\sigma} v\right)=h_{\sigma}\left(f_{\sigma}^{\dagger} f_{\sigma} u, v\right)$. Combined with (4.49), this gives:

$$
f_{\sigma}^{\dagger} f_{\sigma}=\left(A_{\sigma}\right)^{2} \Leftrightarrow A_{\sigma}=\left(f_{\sigma}^{\dagger} f_{\sigma}\right)^{1 / 2} .
$$

Hence the factor $J=\prod_{\sigma \geq 0} \operatorname{det}_{\mathbf{R}} A_{\sigma}^{(-1)^{\sigma+1}}=\prod_{\sigma \geq 0} \operatorname{det}\left(A_{\sigma}\right)^{2(-1)^{\sigma+1}}$ can also be written as:

$$
J=\prod_{\sigma \geq 0} \operatorname{det}\left(f_{\sigma}^{\dagger} f_{\sigma}\right)^{(-1)^{\sigma+1}}
$$

which recovers the prefactor obtained in [9].

As a last observation, we recall that $A_{\sigma}$ are chosen to satisfy relation (4.32). Via relation (4.46), the metrics they determine on $K(\sigma)$ will obey:

$$
g_{\sigma}(c u, c v)=g_{-1-\sigma}(v, u)
$$


where we used the fact that $c$ is anti-unitary:

$$
h_{\sigma}(c u, c v)=h_{-1-\sigma}(v, u)
$$

(this follows from the last of properties (2.22)). As in [9], we introduce antilinear operators $c_{*, \sigma}$ from $H_{\sigma}(\mathcal{H})$ to $H_{-1-\sigma}(\mathcal{H})$ through the conditions:

$$
c_{*, \sigma} f_{\sigma}=f_{-1-\sigma} c_{\sigma}
$$

The relations (4.49) and (4.52) imply:

$$
h_{-1-\sigma}^{H}\left(c_{*, \sigma} u, c_{*, \sigma} v\right)=h_{\sigma}^{H}(v, u),
$$

thereby recovering the constraint on $h_{\sigma}^{H}$ which was used in [9]. In the BV approach, this condition (which is equivalent with $(4.32)$ ), is used to bring the gauge-fixed auxiliary action to the form (4.35).

\subsection{Simplified form of the gauge-fixed action}

Let us consider a correlator of the form:

$$
\left\langle\mathcal{O}_{0} \ldots \mathcal{O}_{n}\right\rangle=\frac{J}{I} \int \mathcal{D}\left[\hat{\phi}_{+}^{M}\right] \mathcal{D}\left[\bar{\phi}^{M}\right] e^{-i \lambda S_{e}\left(\hat{\phi}_{-}=c_{e} d_{e} \bar{\phi}^{M}, \hat{\phi}_{+}=\hat{\phi}_{+}^{M} \oplus \hat{\phi}_{+}^{H}\right)} \mathcal{O}_{1} \ldots \mathcal{O}_{n}
$$

where the observables $\mathcal{O}$ depend only on the physical field. Since $c_{e} d_{e}$ is invertible on the subspace $i m d_{e}^{\dagger}$, one can perform the change of variables $\hat{\phi}_{-}^{M}=c_{e} d_{e} \bar{\phi}^{M}$. This is a linear transformation, under which the path integral measure transforms as:

$$
\mathcal{D}\left[\bar{\phi}^{M}\right]=R \mathcal{D}\left[\hat{\phi}_{-}^{M}\right] .
$$

The prefactor $R$ is clearly independent of all fields (though it does depend on the background superconnection and metric data). This allows us to replace the integral over antighosts with an integral over $\hat{\phi}_{-}^{M} \in \operatorname{imd}_{e}^{\dagger}(s<0)$, up a modification of the normalization factor:

$$
\left\langle\mathcal{O}_{0} \ldots \mathcal{O}_{n}\right\rangle=J \frac{R}{I} \int \prod_{\hat{\phi}^{M} \in i m d_{e}^{\dagger}} e^{-i \lambda S_{e}\left(\hat{\phi}^{M} \oplus \hat{\phi}_{+}^{H}\right)} \mathcal{O}_{1} \ldots \mathcal{O}_{n}
$$

Thus we can ignore the substitution $\phi^{*}=c_{e} d_{e} \bar{\phi}$ in the gauge-fixed action, and simply replace it with the restriction of $S_{e}$ to the subspace $i m d_{e}^{\dagger}$, up to an appropriate shift by $\hat{\phi}_{+}^{H}$.

The quantity $R$ describes the change of the measure under the transformation $\left(c_{e} d_{e}\right)^{-1}: \mathcal{H}_{e}(s<0) \rightarrow \mathcal{H}_{e}(s>0)$. To determine this, we view $\mathcal{H}$ as a real vector space upon restriction of the field of scalars. In this case, one has:

$$
R=\prod_{\sigma>0} \operatorname{det}_{\mathbf{R}}\left((c d)^{t}(c d)\right)^{\frac{(-1)^{\sigma+1}}{2}},
$$


where $\operatorname{det}_{\mathbf{R}}$ denotes the determinant of its argument viewed as a real-linear map and $(c d)^{t}$ is the adjoint of the real-linear operator $c d$ with respect to the induced Euclidean scalar product $(.,)=.\operatorname{Reh}(.,$.$) . Since c d$ is selfadjoint with respect to this product, we have $(c d)^{t}(c d)=c d c d=d^{\dagger} d$. Thus:

$$
R=\prod_{\sigma>0} \operatorname{det}_{\mathbf{R}}\left(d^{\dagger} d\right)^{\frac{(-1)^{\sigma+1}}{2}}=\prod_{\sigma>0} \operatorname{det}\left(d^{\dagger} d\right)^{(-1)^{\sigma+1}}=I
$$

where det stands for the complex determinant and $I$ is the quantity defined in (4.24). In particular, expression (4.58) becomes:

$$
\left\langle\mathcal{O}_{0} \ldots \mathcal{O}_{n}\right\rangle=J \int \prod_{\hat{\phi}^{M} \in i m d_{e}^{\dagger}} e^{-i \lambda S_{e}\left(\hat{\phi}^{M} \oplus \hat{\phi}_{+}^{H}\right)} \mathcal{O}_{1} \ldots \mathcal{O}_{n} .
$$

The formalism encoded by this relation is similar to the description of (ungraded) gaugefixed Chern-Simons theory used in [21]. It has the advantage that its only reference to metric data enters through the particular decomposition of the space of extended fields into subspaces of harmonic, exact and coexact configurations. On the other hand, this description treats the physical field and its ghost/antighost counterparts in a unified manner. This leads to a simple form of the perturbation expansion, as we shall see in Section 6. We stress that this formalism and that described by (4.56) are completely equivalent, being related by a change of variables in the path integral.

\section{The partition function and semiclassical approximation}

Let us consider the path integral ( $\lambda$ is the dimensionless coupling constant):

$$
Z\left(\hat{\phi}^{H}\right)=\frac{J}{I} \int \mathcal{D}\left[\hat{\phi}_{+}^{M}, \bar{\phi}^{M}\right] e^{-i \lambda S_{g f}}=J \int \mathcal{D}\left[\hat{\phi}^{M}\right] e^{-i \lambda S_{e}\left(\hat{\phi}^{M} \oplus \hat{\phi}^{H}\right)} \quad \text { for } \quad \hat{\phi}^{H} \in K_{e} .
$$

In the case when $\hat{\phi}^{H}=\phi^{H}$ (i.e. the harmonic components of ghosts and antighosts are fixed to zero), this quantity can be viewed as the partition function in the background which results from original superconnection by shifting through $\phi^{H}$. In this section, we study the semiclassical (i.e. Gaussian) approximation of (5.1).

\subsection{The semiclassical approximation}

The semiclassical approximation to $Z\left(\hat{\phi}^{H}\right)$ results upon neglecting cubic terms; this amounts to keeping only the kinetic (quadratic) terms of the gauge-fixed action. Since the kinetic term is independent of $\hat{\phi}^{H}$, the resulting quantity $Z_{s c l}$ is also $\hat{\phi}^{H}$-independent and can be computed by setting $\hat{\phi}^{H}=0$. It is instructive to perform the computation in two ways. 


\subsubsection{The first approach}

Let us start from the equation:

$$
Z_{s c l}=\frac{J}{I} \int \mathcal{D}\left[\prod_{\sigma>0} \phi_{\sigma}^{M}\right] \mathcal{D}\left[\prod_{\sigma>0} \bar{\phi}_{\sigma}^{M}\right] e^{-i \lambda S_{g f, k i n}}
$$

where:

$$
S_{g f, k i n}=R e\left[\left\langle\phi_{0}^{M}, d_{e} \phi_{0}^{M}\right\rangle_{e}+\sum_{\sigma>0}\left(\left\langle c_{e} d_{e} \bar{\phi}_{\sigma}^{M}, d_{e} \phi_{\sigma}^{M}\right\rangle_{e}+\left\langle\phi_{\sigma}^{M}, d_{e} c_{e} d_{e} \bar{\phi}_{\sigma}^{M}\right\rangle_{e}\right)\right] .
$$

To perform this Gaussian integral, we consider the scalar product $(\hat{u}, \hat{v})_{e}:=\operatorname{Reh}_{e}(\hat{u}, \hat{v})$ on $\mathcal{H}_{e}$, where $\mathcal{H}$ is viewed as a real vector space by restriction of scalars. This allows us to write:

$$
S_{g f, k i n}=\left(\phi_{0}, c_{e} d_{e} \phi_{0}\right)_{e}+2 \sum_{\sigma>0}(-1)^{\sigma}\left(\bar{\phi}_{\sigma}^{M}, d_{e}^{\dagger} d_{e} \phi_{\sigma}^{M}\right)_{e}
$$

This expression involves the restriction of the Hermitian operator $d^{\dagger} d$ to the subspaces $V_{\sigma}:=i m d^{\dagger} \cap \mathcal{H}(\sigma)=(\text { kerd })^{\perp} \cap \mathcal{H}(\sigma)$ (with $\sigma>0$ ) as well as the restriction of $c d$ to $V_{0}$. Viewing these as real-linear selfadjoint operators, we obtain:

$$
Z_{s c l}=c t \times \frac{J}{I} \operatorname{det}_{\mathbf{R}, s=0}^{\prime}(c d)^{-1 / 2} \prod_{\sigma>0} \operatorname{det}_{\mathbf{R}, s=\sigma}^{\prime}\left(d^{\dagger} d\right)^{(-1)^{\sigma+1}},
$$

where $\operatorname{det}_{\mathbf{R}, s=\sigma}^{\prime}(O)$ stands for the real determinant of an operator $O$ defined on $\mathcal{H}(\sigma)$, after its restriction to the orthogonal complement of its kernel. Using $\operatorname{det}_{\mathbf{R}, s=\sigma}^{\prime}\left(d^{\dagger} d\right)=$ $\operatorname{det}_{s=\sigma}^{\prime}\left(d^{\dagger} d\right)^{2}$ and partially regularizing $\operatorname{det}_{\mathbf{R}, s=0}^{\prime}(c d)$ by replacing the indefinite operator $c d_{s=0}$ with $\left|c d_{s=0}\right|=\sqrt{(c d)_{s=0}^{2}}=\left(d^{\dagger} d_{s=0}\right)^{1 / 2}$, we obtain:

$$
Z_{s c l}=c t \times J \operatorname{det}_{s=0}^{\prime}\left(d^{\dagger} d\right)^{-1 / 2} \prod_{\sigma>0} d e t_{s=\sigma}^{\prime}\left(d^{\dagger} d\right)^{(-1)^{\sigma+1}}
$$

where we used expression (4.24) for $I$. The result (5.6) can be regularized as explained below.

\subsubsection{The second approach}

Expression (5.6) can also be obtained by starting with the equation:

$$
Z_{s c l}=J \int_{\hat{\phi}^{M} \in i m d_{e}^{\dagger}} \mathcal{D}\left[\hat{\phi}^{M}\right] e^{-i \lambda S_{e, k i n}\left(\hat{\phi}^{M}\right)}
$$


where:

$$
\begin{aligned}
S_{e, k i n}\left(\hat{\phi}^{M}\right) & =\operatorname{Re}\left[\left\langle\phi_{0}^{M}, d_{e} \phi_{0}^{M}\right\rangle_{e}+\sum_{\sigma>0}\left(\left\langle\hat{\phi}_{-\sigma}^{M}, d_{e} \hat{\phi}_{\sigma}^{M}\right\rangle_{e}+\left\langle d_{e} \hat{\phi}_{-\sigma}^{M}, \hat{\phi}_{\sigma}^{M}\right\rangle_{e}\right)\right]= \\
& =\left(\phi_{0}^{M}, c_{e} d_{e} \phi_{0}^{M}\right)_{e}+2 \sum_{\sigma>0}(-1)^{\sigma}\left(\hat{\phi}_{-\sigma}^{M}, c_{e} d_{e} \hat{\phi}_{\sigma}^{M}\right)_{e}
\end{aligned}
$$

This gives:

$$
\begin{aligned}
Z_{s c l} & =c t \times J \operatorname{det}_{\mathbf{R}, s=0}^{\prime}(c d)^{-1 / 2} \prod_{\sigma>0} \operatorname{det}_{\mathbf{R}, s=\sigma}^{\prime}\left(d^{\dagger} d\right)^{\frac{(-1)^{\sigma+1}}{2}}= \\
& =c t \times J d e t_{s=0}^{\prime}\left(d^{\dagger} d\right)^{-1 / 2} \prod_{\sigma>0} \operatorname{det}_{s=\sigma}^{\prime}\left(d^{\dagger} d\right)^{(-1)^{\sigma+1}},
\end{aligned}
$$

therefore recovering (5.6).

\subsubsection{Regularization}

Expression (5.6) is of course ill defined. This is cured in standard manner by using zeta-function regularization. Given a positive elliptic operator $O$ acting on sections of the bundle $\mathcal{V}$, recall that its zeta function is defined through the expansion:

$$
\zeta_{O}(z)=\sum_{\lambda} \frac{n_{\lambda}}{\lambda^{z}}
$$

where $\lambda$ are the distinct eigenvalues of $O$ and $n_{\lambda}$ are their multiplicities. The series (5.10) converges for large enough $R e z$, and admits a continuation to a meromorphic function defined in the complex plane, which is regular at the origin. If $\zeta_{O}^{\prime}(z):=\frac{d \zeta_{O}(z)}{d z}$ denotes its derivative, then one defines the regularized determinant through:

$$
\operatorname{det}^{r e g}(O):=e^{-\zeta_{O}^{\prime}(0)}
$$

Upon regularizing in this manner and using (4.51), the result (5.6) becomes:

$$
Z_{s c l}=C T\left(L, A_{0}\right)^{-1} \prod_{\sigma \geq 0}\left[\operatorname{det}\left(f_{\sigma}^{\dagger} f_{\sigma}\right)\right]^{(-1)^{\sigma+1}}
$$

where $C$ is a complex constant and we defined the graded Ray-Singer torsion by:

$$
T(L, A):=\operatorname{det}_{s=0}^{\prime}{ }_{s=g}^{r e g}(c d)^{1 / 2} \prod_{\sigma>0} \operatorname{det}_{s=\sigma}^{\prime}{ }_{s=\sigma}^{r e g}\left(d^{\dagger} d\right)^{(-1)^{\sigma}} .
$$

Equations (5.12) and (5.13) can be recognized as the expressions derived in [9], which were obtained in that paper by using the method of resolvents developed in $[24,25,26]$. As expected from the general remarks of [27, 28], this method indirectly implements the effect of ghosts and antighosts, which is apparent from our computations above. As explained in [9], the quantity (5.13) can be used to define a 'graded Ray-Singer norm', which is a topological invariant-a result which generalizes the well-known construction of $[39,40,41]$. 


\section{The effective potential}

Considering the path integral (5.1), we define the extended potential through:

$$
e^{-i \lambda W_{e}\left(\hat{\phi}^{H}\right)}=\frac{Z\left(\hat{\phi}^{H}\right)}{Z(0)}=\frac{\int \mathcal{D}\left[\hat{\phi}^{M}\right] e^{-i \lambda S_{e}\left(\hat{\phi}^{M} \oplus \hat{\phi}^{H}\right)}}{\int \mathcal{D}\left[\hat{\phi}^{M}\right] e^{-i \lambda S_{e}\left(\hat{\phi}^{M}\right)}}
$$

While this definition makes sense for arbitrary $\hat{\phi}^{H}$, the quantity $W_{e}$ has a direct physical interpretation only if we restrict to physical field shifts, $\hat{\phi}^{H}=\hat{\phi}_{0}^{H}=\phi^{H}$. Therefore, we define the physical potential $W$ by:

$$
W\left(\phi^{H}\right)=e v_{G}\left(W_{e}\left(\phi^{H} \otimes 1_{G}\right)\right) \quad \text { for } \phi^{H} \in K^{1} .
$$

As we shall see below, this is precisely the potential whose tree-level approximation was discussed in a slightly more naive language in [8]. Note that (6.1) provides a nonperturbative definition of the potential, and in particular gives a prescription for its perturbative expansion to all loop orders.

\subsection{Perturbative expansion}

The perturbative expansion of $W_{e}$ is obtained as follows. First, we note that:

$$
\begin{aligned}
& S_{e}\left(\hat{\phi}^{M} \oplus \hat{\phi}^{H}\right)=S_{e}\left(\hat{\phi}^{M}\right)+S_{e, I}\left(\hat{\phi}^{M}, \hat{\phi}^{H}\right), \text { with : } \\
& S_{e, I}\left(\hat{\phi}^{M}, \hat{\phi}^{H}\right)=\frac{1}{3}\left\langle\hat{\phi}^{H}, \hat{\phi}^{H} * \hat{\phi}^{H}\right\rangle_{e}+\left\langle\hat{\phi}^{H}, \hat{\phi}^{M} * \hat{\phi}^{M}\right\rangle_{e}+\left\langle\hat{\phi}^{M}, \hat{\phi}^{H} * \hat{\phi}^{H}\right\rangle_{e},
\end{aligned}
$$

where we used the fact that $\hat{\phi}^{H}$ brings no contribution to the kinetic term:

$$
\left\langle\hat{\phi}^{H}, d_{e} \hat{\phi}\right\rangle_{e}=\left\langle\hat{\phi}, d_{e} \hat{\phi}^{H}\right\rangle_{e}=0
$$

and where $S_{e}\left(\hat{\phi}^{M}\right)=\frac{1}{2}\left\langle\hat{\phi}^{M}, d_{e} \hat{\phi}^{M}\right\rangle_{e}+\frac{1}{3}\left\langle\hat{\phi}^{M}, \hat{\phi}^{M} * \hat{\phi}^{M}\right\rangle_{e}$. Substitution of (6.3) in (6.1) gives:

$$
e^{-i \lambda W_{e}\left(\hat{\phi}^{H}\right)}=\frac{\int \mathcal{D}\left[\hat{\phi}^{M}\right] e^{-i \lambda\left[S_{e}\left(\hat{\phi}^{M}\right)+S_{e, I}\left(\hat{\phi}^{M}, \hat{\phi}^{H}\right)\right]}}{\int \mathcal{D}\left[\hat{\phi}^{M}\right] e^{-i \lambda S_{e}\left(\hat{\phi}^{M}\right)}} .
$$

This leads to a perturbative series for $W_{e}$ upon expanding the exponential terms in $S_{e, I}\left(\hat{\phi}^{M}, \hat{\phi}^{H}\right)$. Since only $\hat{\phi}^{M}$ has a kinetic term, and since the path integral in the numerator is performed over this component only, this leads to Feynman integrals in which $\hat{\phi}^{H}$ are treated as (amputated) external insertions, and only $\hat{\phi}^{M}$ propagate. Feynman diagrams are built out of the vertices and propagator depicted in figure 3 . We note that this description automatically takes ghosts and antighosts into account. In our formalism, their contributions are described by the non-physical components $\hat{\phi}_{\sigma}^{M}$ $(\sigma \neq 0)$ of $\hat{\phi}^{M}$. 

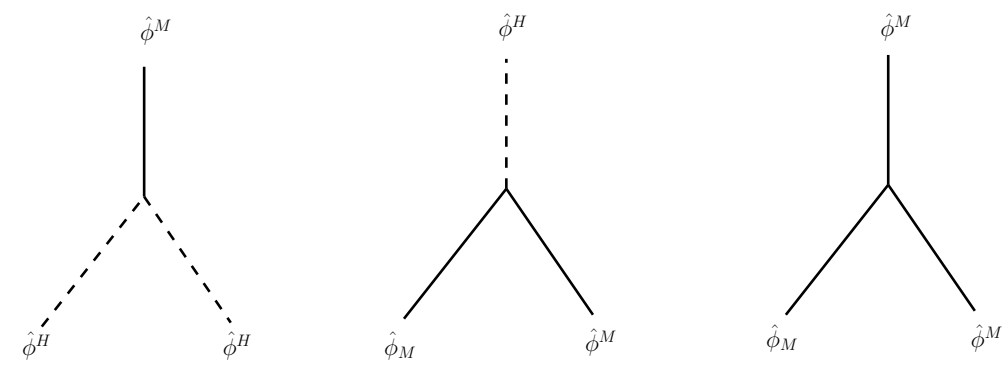

Figure 3: The vertices and propagator involved in the expansion of $W_{e}$.

\subsection{The propagator of nonzero-modes}

The propagator $U_{e}$ of $\hat{\phi}^{M}$ can be computed in standard manner. Proceeding as usual, we consider the free generating functional:

$$
\begin{aligned}
Z_{\text {free }}[\hat{L}] & =J \int \mathcal{D}\left[\hat{\phi}^{M}\right] e^{-i \lambda \operatorname{Re}\left[\left\langle\hat{\phi}^{M}, d_{e} \hat{\phi}^{M}\right\rangle_{e}+\left\langle\hat{L}, \hat{\phi}^{M}\right\rangle_{e}\right]} \\
& =J \int \mathcal{D}\left[\hat{\phi}^{M}\right] e^{-i \lambda \operatorname{Re}\left[\left\langle\hat{\phi}^{M}, d_{e} \hat{\phi}^{M}\right\rangle_{e}+\left\langle\pi_{d_{e}} \hat{L}, \hat{\phi}^{M}\right\rangle_{e}\right]},
\end{aligned}
$$

with the external current ${ }^{3} \hat{L} \in \mathcal{H}_{e}^{0}$. To arrive at the second form, we used the last of properties (2.24) and the fact that $\hat{\phi}^{M}$ belongs to $i m d_{e}^{\dagger}$ (together, these imply that the component of $\hat{L}$ along $k e r d_{e}^{\dagger}=K_{e} \oplus i m d_{e}^{\dagger}$ does not contribute to the external coupling term).

The dependence of $\hat{L}$ can be extracted by shifting $\hat{\phi}^{M} \rightarrow \hat{\phi}^{M}-\frac{1}{2} d_{e}^{-1} \pi_{d_{e}} \hat{L}$, where $d_{e}^{-1}$ is the inverse of the operator $d_{e}: i m d_{e}^{\dagger} \rightarrow i m d_{e}$. This gives:

$$
\left\langle\hat{\phi}^{M}, d_{e} \hat{\phi}^{M}\right\rangle_{e}+\left\langle\pi_{d_{e}} \hat{L}, \hat{\phi}^{M}\right\rangle_{e} \rightarrow\left\langle\hat{\phi}^{M}, d_{e} \hat{\phi}^{M}\right\rangle_{e}-\frac{1}{4}\left\langle\pi_{d_{e}} \hat{L}, d_{e}^{-1} \pi_{d_{e}} \hat{L}\right\rangle_{e}+\frac{1}{2} T
$$

where:

$$
T=\left\langle\hat{\phi}^{M}, \pi_{d_{e}} \hat{L}\right\rangle_{e}-\left\langle d_{e}^{-1} \pi_{d_{e}} \hat{L}, d_{e} \hat{\phi}^{M}\right\rangle_{e}=0
$$

since $\left\langle d_{e}^{-1} \pi_{d_{e}} \hat{L}, d_{e} \hat{\phi}^{M}\right\rangle_{e}=\left\langle d_{e} d_{e}^{-1} \pi_{d_{e}} \hat{L}, \hat{\phi}^{M}\right\rangle_{e}=\left\langle\pi_{d_{e}} \hat{L}, \hat{\phi}^{M}\right\rangle_{e}=\left\langle\hat{\phi}^{M}, \pi_{d_{e}} \hat{L}\right\rangle_{e}$. Thus:

$$
Z_{\text {free }}[\hat{L}]=Z_{s c l} e^{\frac{i \lambda}{4} R e\left\langle\pi_{d_{e}} \hat{L}, d_{e}^{-1} \pi_{d_{e}} \hat{L}\right\rangle_{e}},
$$

where:

$$
Z_{s c l}=J \int \mathcal{D}\left[\hat{\phi}^{M}\right] e^{-i \lambda \operatorname{Re}\left\langle\hat{\phi}^{M}, d_{e} \hat{\phi}^{M}\right\rangle_{e}}
$$

\footnotetext{
${ }^{3}$ Note that we do not restrict the current $\hat{L}$ to belong to $i m d_{e}^{\dagger}$; this allows us to describe propagator insertions at vertices induced by the last two terms of $S_{e, I}$ in eq. (6.3).
} 
Consider the extended propagator:

$$
U_{e}:=d_{e}^{-1} \pi_{d_{e}}=\frac{1}{\Delta_{e}} d_{e}^{\dagger}
$$

Then: $\left\langle\pi_{d_{e}} \hat{L}, d_{e}^{-1} \pi_{d_{e}} \hat{L}\right\rangle_{e}=\left\langle\hat{L}, U_{e} \hat{L}\right\rangle_{e}$ by the last property in $(2.24)$, since $i m U_{e}=i m d^{\dagger}$, and since $d_{e}^{\dagger} \hat{L}=d_{e}^{\dagger} \pi_{d_{e}} \hat{L}$. This allows us to re-write (6.9) as:

$$
Z_{\text {free }}[\hat{L}]=Z_{s c l} e^{\frac{i \lambda}{4} R e\left\langle\hat{L}, U_{e} \hat{L}\right\rangle_{e}} .
$$

Vertex insertions in the perturbative expansion of $W_{e}$ are now obtained by cubic functional differentiation of $Z_{\text {free }}[\hat{L}]$ with respect to $\hat{L}$. This also brings down insertions of the extended propagator $U_{e}$.

Since $d_{e}=d \otimes i d_{G}$ and $\Delta_{e}=\Delta \otimes i d_{G}, U_{e}$ is related to the propagator $U$ of Subsection 2.2.2 by:

$$
U_{e}=U \otimes i d_{G}
$$

The operators $U_{e}$ and $U$ have ghost degree $s=1$, and decompose as:

$$
U_{e}=\oplus_{\sigma} U_{e}^{\sigma}, \quad U=\oplus_{\sigma} U_{\sigma}
$$

where $U_{\sigma}$ are linear operators from $\mathcal{H}(\sigma)$ to $\mathcal{H}(\sigma+1)$, while $U_{e}^{\sigma}=U_{\sigma} \otimes i d_{G}$ are operators from $\mathcal{H}_{e}(\sigma)$ to $\mathcal{H}_{e}(\sigma+1)$. The operators $U_{\sigma}$ play the role of propagators for the various components of $\hat{\phi}$, so that $U$ contains both the physical propagator and the propagators of ghosts and antighosts. In this formulation, $U$ does not conserve the ghost number $s$ (since the kinetic operators $d_{e}$ and $d$ do not). As in the usual ChernSimons case, conservation of ghost number can be achieved in the equivalent formalism which describes the path integral directly in terms of ghosts and antighosts (related to the present description by the change of variables explained in Subsection 4.4.)

\subsection{Feynman rules}

The Feynman rules can now be constructed in standard manner. In our formulation, they are virtually identical with the Feynman rules of usual Chern-Simons theory. As in that case, the vertices are only cyclically symmetric. This implies that the correspondence between Feynman integrals and graphs depends on an orientation of the plane (in which the graph sits). This is not surprising since our theories can be interpreted as string field theories of oriented topological strings [6]. The orientation is fixed once we decide what correlation function corresponds to a graph with one vertex and three external legs and, of course, the same orientation has to be used at all vertices of more complicated graphs. 


\subsection{Tree-level products}

The perturbative expansion of $W_{e}$ can be arranged according to the number of loops. In this subsection, we are interested in the tree-level approximation $W_{e}^{\text {tree }}$, which comes from graphs without internal circuits. An example of such a graph is shown in figure 4 .

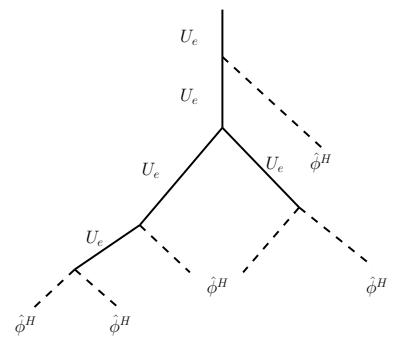

Figure 4: Example of a tree-level graph contributing to the perturbative expansion of $W_{e}$

While perturbative computation of the effective potential will generally require calculation of scattering amplitudes, the tree-level approximation $W_{e}^{\text {tree }}$ can also be obtained by using the procedure discussed in Appendix B of [8]. The only difference between our situation and the treatment given there is that we are considering the extended field $\hat{\phi}$, rather than the classical field $\phi$.

Before we give the result, let us outline the construction. We begin by computing the functional derivative of the extended potential $W_{e}$ using definition (6.1). The result is that this is given by the expectation value of the derivative of $S_{e}\left(\hat{\phi}^{M} \oplus \hat{\phi}^{H}\right)$ with respect to $\hat{\phi}^{H}$. At this point we encounter the first similarity with [8]. Using the properties of the extended product and extended bilinear form it is easy to see that the derivative of the extended action with respect to $\hat{\phi}^{H}$ is obtained from the derivative of the unextended action with respect to $\phi^{H}$ by replacing the unextended fields with extended fields and the product $\bullet$ with $*$.

Since we are interested in the tree level potential, we can use the saddle point approximation and find that we need to evaluate the derivative of the extended action on a solution of the extended equation of motion $d \hat{\phi}^{M}+\left(\hat{\phi}^{M}+\hat{\phi}^{H}\right) *\left(\hat{\phi}^{M}+\hat{\phi}^{H}\right)=0$ (the critical point equations for $S_{e}\left(\hat{\phi}^{M} \oplus \hat{\phi}^{H}\right)$ with respect to $\left.\hat{\phi}^{M}\right)$. Since the latter is obtained from the unextended equation of motion by the replacement described above, so is the corresponding solution. Hence the tree level products of the extended theory are obtained from those of the unextended one by similar replacements. Accordingly, we can use Appendix B of [8] to conclude that the extended tree-level potential can be 
written in the form:

$$
W_{e}^{\text {tree }}=-\sum_{n \geq 2} \frac{(-1)^{n(n+1) / 2}}{n+1} \lambda^{n-2}\left\langle\hat{\phi}^{H}, r_{n}^{e}\left(\left(\hat{\phi}^{H}\right)^{\otimes n}\right)\right\rangle_{e}
$$

where:

$$
r_{n}^{e}\left(\left(\hat{\phi}^{H}\right)^{\otimes n}\right)=P_{e} \lambda_{n}^{e}\left(\left(\hat{\phi}^{H}\right)^{\otimes n}\right)
$$

and $P_{e}$ is the projector on $K_{e}$. Exactly as in [8], the products $\lambda_{n}^{e}$ satisfy the recurrence relations:

$$
\begin{aligned}
\lambda_{2}^{e}\left(\left(\hat{\phi}^{H}\right)^{\otimes 2}\right) & =\hat{\phi}^{K} * \hat{\phi}^{K} \\
\lambda_{n}^{e}\left(\left(\hat{\phi}^{H}\right)^{\otimes n}\right) & =(-1)^{n-1} \hat{\phi}^{H} * U_{e} \lambda_{n-1}^{e}\left(\left(\hat{\phi}^{H}\right)^{\otimes(n-1)}\right)+(-1)^{n-1} U_{e} \lambda_{n-1}^{e}\left(\left(\hat{\phi}^{H}\right)^{\otimes(n-1)}\right) * \hat{\phi}^{H} \\
& -\sum_{\substack{l+m=n \\
l, m \geq 2}}(-1)^{m l} U_{e} \lambda_{l}^{e}\left(\left(\hat{\phi}^{H}\right)^{\otimes l}\right) * U_{e} \lambda_{m}^{e}\left(\left(\hat{\phi}^{H}\right)^{\otimes m}\right) \text { for } n \geq 3 .
\end{aligned}
$$

The extended potential constructed here depend on physical fields as well as ghosts and antighosts. To isolate the potential for physical fields we particularize to harmonic shifts of the form $\hat{\phi}^{K}=\phi^{K} \otimes 1_{G}$, where $\phi_{K} \in \mathcal{H}^{1}$. As explained in Subsection 3.1., the products $*$ and $\bullet$ agree for such fields - in fact, we have $\left(u \otimes 1_{G}\right) *\left(v \otimes 1_{G}\right)=(u \bullet v) \otimes 1_{G}$, since the unit $1_{G}$ of the Grassmann algebra is even. Combining this with the properties $U_{e}=U \otimes i d_{G}, P_{e}=P \otimes i d_{G}$, we obtain:

$$
\lambda_{n}^{e}\left(\left(\phi^{H} \otimes 1_{G}\right)^{\otimes n}\right)=\lambda_{n}\left(\left(\phi^{H}\right)^{\otimes n}\right) \otimes 1_{G} \quad, \quad r_{n}^{e}\left(\left(\phi^{H} \otimes 1_{G}\right)^{\otimes n}\right)=r_{n}\left(\left(\phi^{H}\right)^{\otimes n}\right) \otimes 1_{G} .
$$

These relations follow by a simple induction argument, upon using the fact that $\phi^{H}$ has even Grassmannality and the definition (3.4) of the extended product.

This allows us to recover the tree-level potential $W^{\text {tree }}$ constructed in [8]:

$$
W_{e}^{\text {tree }}\left(\phi^{H} \otimes 1_{G}\right)=W^{\text {tree }}\left(\phi^{H}\right) \otimes 1_{G},
$$

where we used definition (3.6) of the extended bilinear form.

\section{Conclusions}

We discussed gauge-fixing of graded Chern-Simons field theories. Upon choosing a 'universal' delta-function gauge fermion, we showed that the resulting gauge-fixed action leads to a formalism which is a graded version of the description given in [21]. This leads to a simple expression for the propagator of nonzero-modes, and allows for a systematic treatment of the semiclassical approximation. In particular, we re-derived the semiclassical partition function of [9] within the BV formalism, obtaining complete 
agreement with the results predicted by the method of resolvents. Finally, we discussed the perturbative expansion of the effective potential for zero modes, and provided the BV justification of the construction used in [8].

The current work (and, more generally, our interest in graded Chern-Simons theories) is motivated by their role in a string-field theoretic description of topological D-branes in Calabi-Yau compactifications. In the present context, this arises when the 3-manifold $L$ is a special Lagrangian 3-cycle of a Calabi-Yau threefold. Regarding this connection, we mention that there exists a $\mathrm{B}$-model version of our theories [7], which is in many ways extremely similar; we chose to focus on the A-model mostly due to its nontrivial topological implications.

As discussed in [8], the graded Chern-Simons description allows for a natural definition of the moduli space of graded D-branes, which does not seem to admit a simple treatment in other approaches. From that perspective, it is interesting to consider the role of our potential and semiclassical approximation at the level of the associated triangulated categories $[6,7]$. This and related issues are currently under investigation.

\section{Acknowledgments}

We are indebted to M. Rocek for support and interest in our work. C.I. L. thanks Rutgers University (where part of this paper was completed) for hospitality and providing excellent conditions. The present work was supported by the Research Foundation under NSF grants PHY-9722101, NSFPHY00-98395 (6T) and by the DOE grant 91ER40618 (3N).

\section{A. A weighted gauge}

One can choose an alternate gauge fixing fermion for our systems and show that that the extra ghosts decouple in the gauge-fixed action; the corresponding family of weighted gauges recovers the Landau gauge of Section 4 in an appropriate limit. The present appendix gives a general discussion of this gauge as well as an independent treatment for an example, showing how the weighted gauge-fixing procedure can be recovered by more elementary means in a particular case. For simplicity, we start once again with the acyclic case.

\section{A.1 The acyclic case}

For an acyclic background, the weighted gauge-fixing fermion has the form:

$$
\Psi_{N}=\operatorname{Re} \int_{L} s t r_{e} \sum_{k \geq 0}\left[\sum_{\sigma \geq 2 k} \bar{\phi}_{\sigma+1}^{N}[k] * d_{e} c_{e} \phi_{\sigma}^{N}[k]+\sum_{\sigma \geq 2 k+1} \phi_{\sigma+1}^{N}[k+1] * d_{e} c_{e} \bar{\phi}_{\sigma}^{N}[k]\right.
$$




$$
\begin{aligned}
& +\Xi \sum_{\sigma \geq 2 k} \bar{\phi}_{\sigma+2}^{N}[k] * c_{e} \pi_{\sigma+2}^{N}[k+1]+\Xi \sum_{\sigma \geq 2 k+1} \phi_{\sigma+1}^{N}[k+1] * c_{e} \bar{\pi}_{\sigma+1}^{N}[k] \\
& \left.+\Xi \bar{\phi}^{N}{ }_{2 k+1}[k] * c_{e} \bar{\pi}_{2 k+1}^{N}[k]\right]
\end{aligned}
$$

where the positive constant $\Xi$ is the weight. The antifields are now eliminated through the equations:

$$
\begin{aligned}
\phi_{\sigma}^{N *}[k] & =c_{e} d_{e} \bar{\phi}_{\sigma+1}^{N}[k]-d_{e} c_{e} \bar{\phi}_{\sigma-1}^{N}[k-1]-\Xi c_{e} \bar{\pi}_{\sigma}^{N}[k-1] \\
\bar{\phi}_{\sigma}^{N^{*}}[k] & =c_{e} d_{e} \phi_{\sigma+1}^{N}[k+1]-d_{e} c_{e} \phi_{\sigma-1}^{N}[k]-\Xi c_{e} \pi_{\sigma}^{N}[k+1]-\Xi \delta_{\sigma, 2 k+1} c_{e} \bar{\pi}_{2 k+1}^{N}[k] .
\end{aligned}
$$

For $k=0$, the first relation gives:

$$
\phi_{\sigma}^{N *}=c_{e} d_{e} \bar{\phi}_{\sigma+1}^{N}
$$

which reproduces the gauge-fixing condition of Section 4. Note that the auxiliary fields $\pi^{N}$ and $\bar{\pi}^{N}$ will not appear in $S_{e, g f}$ once we replace the antifields by the equations above. Indeed, the only antifields appearing in $S_{e}$ are $\phi_{\sigma}^{N *}[0]$ and they do not depend on $\pi^{N}$ and $\bar{\pi}^{N}$.

Substituting (A.2) in the auxiliary action and using the equations of motion to eliminate the auxiliary fields $\pi^{N}$ and $\bar{\pi}^{N}$, we find:

$$
\begin{aligned}
& S_{a u x, g f}=\frac{1}{4 \Xi} R e \sum_{k \geq 0}\left\langle c_{e} d_{e} \phi_{2(k+1)}^{N}[k+1]-d_{e} c_{e} \phi_{2 k}^{N}[k], d_{e} \phi_{2(k+1)}^{N}[k+1]-c_{e} d_{e} c_{e} \phi_{2 k}^{N}[k]\right\rangle_{e} \\
& +\frac{1}{2 \Xi} R e \sum_{k \geq 1} \sum_{\sigma \geq 2 k}(-1)^{\sigma+1}\left\langle c_{e} d_{e} \phi_{\sigma+1}^{N}[k]-d_{e} c_{e} \phi_{\sigma-1}^{N}[k-1],(-1)^{\sigma+1} d_{e} \bar{\phi}_{\sigma+1}^{N}[k]+c_{e} d_{e} c_{e} \bar{\phi}_{\sigma-1}^{N}[k-1]\right\rangle_{e} .
\end{aligned}
$$

Integration by parts gives the equivalent form:

$$
\begin{aligned}
S_{a u x, g f}= & -\frac{1}{4 \Xi} R e\left\langle\phi_{0}^{N}[0], c_{e} d_{e} c_{e} d_{e} c_{e} \phi_{0}^{N}[0]\right\rangle_{e}-\frac{1}{4 \Xi} R e \sum_{k \geq 1}\left\langle\phi_{2 k}^{N}[k],\left(d c d+c_{e} d_{e} c_{e} d_{e} c_{e}\right) \phi_{2 k}^{N}[k]\right\rangle_{e} \\
& +\frac{1}{2 \Xi} R e \sum_{k \geq 1} \sum_{\sigma \geq 2 k+1}\left\langle\phi_{\sigma}^{N}[k],\left[d_{e} c_{e} d_{e}+(-1)^{\sigma} c_{e} d_{e} c_{e} d_{e} c_{e}\right] \bar{\phi}_{\sigma}^{N}[k]\right\rangle_{e} \\
& +\frac{1}{2 \Xi} \operatorname{Re} \sum_{\sigma \geq 2}(-1)^{\sigma}\left\langle\phi_{\sigma}^{N}[0], c_{e} d_{e} c_{e} d_{e} c_{e} \bar{\phi}_{\sigma}^{N}[0]\right\rangle_{e},
\end{aligned}
$$

where we used $c_{e}^{2} \hat{u}=(-1)^{g(\hat{u})} \hat{u}$. This expression shows that the extraghosts are free and thus can be integrated out. This produces an appropriate prefactor in the path integral measure and allows us to replace $S_{a u x}$ by its first term:

$$
S_{a u x, f d} \equiv-\frac{1}{4 \Xi} \operatorname{Re}\left\langle\phi_{0}^{N}, c_{e} d_{e} c_{e} d_{e} c_{e} \phi_{0}^{N}\right\rangle_{e}=-\frac{1}{4 \Xi} \operatorname{Re}\left\langle\phi_{0}^{N}, c_{e} d_{e} d_{e}^{\dagger} \phi_{0}^{N}\right\rangle_{e}=\frac{1}{4 \Xi} \operatorname{Re} h_{e}\left(d_{e}^{\dagger} \phi_{0}^{N}, d_{e}^{\dagger} \phi_{0}^{N}\right)
$$

Hence the result of gauge-fixing can be described through the action:

$$
S_{g f}^{\Xi}=S_{e}\left(\phi_{\sigma}^{N *}=c_{e} d_{e} \bar{\phi}_{\sigma+1}^{N}, \phi_{\sigma}^{N}\right)+\frac{1}{4 \Xi} \operatorname{Reh}_{e}\left(d_{e}^{\dagger} \phi_{0}^{N}, d_{e}^{\dagger} \phi_{0}^{N}\right)
$$


The gauge of Section 4 is now obtained in the Landau-type limit $^{4} \Xi \rightarrow 0$. As usual, this can be implemented at the level of the action by dropping $S_{\text {aux }}$ and keeping in mind that all propagators need to be transverse. Alternately, one can take this limit at the level of Feynman rules (in which case transverse propagators will be produced automatically). This reproduces the Landau gauge.

\section{A.2 Inclusion of zero-modes}

In the presence of zero-modes, we supplement the gauge-fixing fermion by adding the harmonic piece:

$$
\begin{aligned}
\Psi_{K} & =\operatorname{Re} \int_{L} s t r_{e} \sum_{k \geq 0}\left(\sum_{\sigma \geq 2 k}\left(c_{e} \bar{\mu}_{\sigma}^{K}[k]\right) * A_{\sigma}[k]^{1 / 2} \phi_{\sigma}^{K}[k]+\sum_{\sigma \geq 2 k+1}\left(c_{e} \mu_{\sigma}^{K}[k]\right) * A_{\sigma}[k]^{1 / 2} \bar{\phi}_{\sigma}^{K}[k]\right) \\
& +\Xi \operatorname{Re} \int_{L} \operatorname{str} \sum_{k \geq 0}\left(\sum_{\sigma \geq 2 k} A_{\sigma+2}[k]^{1 / 2} \bar{\phi}_{\sigma+2}^{K}[k] * c_{e} \pi_{\sigma+2}^{K}[k+1]+\right. \\
& \left.+\sum_{\sigma \geq 2 k+1} A_{\sigma+1}[k+1]^{1 / 2} \phi_{\sigma+1}^{K}[k+1] * c_{e} \bar{\pi}_{\sigma+1}^{K}[k]+A_{2 k+1}[k]^{1 / 2} \bar{\phi}_{2 k+1}^{K}[k] * c_{e} \bar{\pi}_{2 k+1}^{K}[k]\right)
\end{aligned}
$$

where $A_{\sigma}[k]$ are, as in Section 4.2.2, some strictly positive operators on $K(\sigma)$. This fixes the harmonic components of antifields to the values:

$$
\begin{gathered}
\phi_{\sigma}^{K *}[k]=\frac{\delta \Psi}{\delta \phi_{\sigma}^{K}[k]}=A_{\sigma}[k]^{1 / 2} c_{e} \bar{\mu}_{\sigma}^{K}[k]+\Xi \delta\left(\begin{array}{c}
k \geq 1 \\
\sigma \geq 2 k)
\end{array}\right) A_{\sigma}[k]^{1 / 2} c_{e} \bar{\pi}_{\sigma}^{K}[k-1] \\
\bar{\phi}_{\sigma}^{K *}[k]=\frac{\delta \Psi}{\delta \bar{\phi}_{\sigma}^{K}[k]}=A_{\sigma}[k]^{1 / 2} c_{e} \mu_{\sigma}^{K}[k]+\Xi \delta\left(\begin{array}{c}
k \geq 0 \\
k \geq 2 k+2
\end{array}\right) A_{\sigma}[k]^{1 / 2} c_{e} \pi_{\sigma}^{K}[k+1] \\
+\Xi \delta_{\sigma, 2 k+1} \delta k \geq 0 A_{2 k+1}[k]^{1 / 2} c_{e} \bar{\pi}_{2 k+1}^{K}[k] \\
\mu_{\sigma}^{K *}[k]=\frac{\delta \Psi}{\delta \mu_{\sigma}^{K}[k]}=c_{e} A_{\sigma}[k]^{1 / 2} \bar{\phi}_{\sigma}^{K}[k] ; \quad \bar{\mu}_{\sigma}^{K *}[k]=\frac{\delta \Psi}{\delta \bar{\mu}_{\sigma}^{K}[k]}=c_{e} A_{\sigma}[k]^{1 / 2} \phi_{\sigma}^{K}[k] .
\end{gathered}
$$

Substituting them in the auxiliary action gives:

$$
\begin{aligned}
S_{a u x}=\operatorname{Re} \int_{L} \operatorname{str}( & \left(\sum_{k \geq 0} \sum_{\sigma \geq 2 k} \lambda_{\sigma}^{K}[k] * A_{\sigma}[k] c_{e} \phi_{\sigma}^{K}[k]\right. \\
& \left.+\sum_{k \geq 0} \sum_{\sigma \geq 2 k+1} \bar{\lambda}_{\sigma}^{K}[k] * A_{\sigma}[k] c_{e} \bar{\phi}_{\sigma}^{K}[k]\right) \\
+ & \operatorname{Re} \int_{L} \operatorname{str}\left(\sum_{k \geq 1} \sum_{\sigma \geq 2 k} \pi_{\sigma}^{K}[k] * A_{\sigma}[k] c_{e}\left(\bar{\mu}_{\sigma}^{K}[k]+\Xi \bar{\pi}_{\sigma}^{K}[k-1]\right)\right.
\end{aligned}
$$

\footnotetext{
${ }^{4}$ Since our systems are defined on the compact manifold $L$, we do not have to worry about infrared singularities when taking this limit. Compacity of $L$ acts as an automatic infrared regulator, which we never have to remove.
} 


$$
\begin{aligned}
& +\sum_{k \geq 0} \sum_{\sigma \geq 2 k+2} \bar{\pi}_{\sigma}^{K}[k] * A_{\sigma}[k] c_{e}\left(\mu_{\sigma}^{K}[k]+\Xi \pi_{\sigma}^{K}[k+1]\right) \\
& \left.+\sum_{k \geq 0} \bar{\pi}_{2 k+1}^{K}[k] * A_{\sigma}[k] c_{e}\left(\mu_{2 k+1}^{K}[k]+\Xi \bar{\pi}_{2 k+1}^{K}[k]\right)\right) .
\end{aligned}
$$

The integral over $\lambda$ and $\bar{\lambda}$ gives the same result as before. For the remaining variables, we first integrate over $\pi[k]$ and find

$$
\prod_{\substack{k \geq 1 \\ \sigma \geq 2 k}} \delta\left(A_{\sigma}[k] c_{e}\left(\bar{\mu}_{\sigma}^{K}[k]+\Xi \bar{\pi}_{\sigma}^{K}[k-1]\right)+\Xi c_{e} A_{\sigma}[k] \bar{\pi}_{\sigma}[k-1]\right)
$$

Now integration over $\bar{\mu}$ produces the first factor in (4.37). The remaining integrals produce the second factor in that equation.

\section{A.3 An example}

Let us consider an example related to the 'D-brane pairs of unit relative grade', which were discussed from various points of view in [29, 8, 9]. For the reader's convenience, we give a direct construction of the gauge-fixing fermion discussed on general grounds above. For simplicity, we shall assume an acyclic background.

The setup consists of two graded flat bundles (of equal ranks) $E_{a}$ and $E_{b}$ on $L$, whose grades differ by one: $\operatorname{grade}(b)=\operatorname{grade}(a)+1$. A generic field configuration degree $|u|=n$ is represented by the matrix

$$
u=\left[\begin{array}{ll}
u_{a a}^{(n)} & u_{b a}^{(n+1)} \\
u_{a b}^{(n-1)} & u_{b b}^{(n)}
\end{array}\right]
$$

with:

$$
\begin{gathered}
u_{a a}^{(1)} \in \operatorname{Hom}^{1}(a, a)=\Omega^{1}\left(\operatorname{End}\left(E_{a}\right)\right), u_{b b}^{(1)} \in \operatorname{Hom}^{1}(b, b)=\Omega^{1}\left(\operatorname{End}\left(E_{b}\right)\right) \\
u_{a b}^{(1-n)} \in \operatorname{Hom}^{1}(a, b)=\Omega^{1-n}\left(\operatorname{Hom}_{(}\left(E_{a}, E_{b}\right)\right), u_{b a}^{(1+n)} \in \operatorname{Hom}^{1}(b, a)=\Omega^{1+n}\left(\operatorname{Hom}\left(E_{b}, E_{a}\right)\right)
\end{gathered}
$$

where the superscripts in round brackets indicate form rank. This is the notation used in [29]. The physical fields $\phi_{0}[0]$ and first and second generation ghosts $\phi_{1}[0]$ and $\phi_{2}[0]$ have the form:

$$
\phi_{0}[0]=\left(\begin{array}{ll}
\phi^{(1)} & \phi^{(2)} \\
\phi^{(0)} & \phi^{(1)}
\end{array}\right) \quad, \quad \phi_{1}[0]=\left(\begin{array}{cc}
c_{1}^{(0)} & c_{1}^{(1)} \\
0 & c_{1}^{\prime(0)}
\end{array}\right) \quad, \quad \phi_{2}[0]=\left(\begin{array}{cc}
0 & c_{2}^{(0)} \\
0 & 0
\end{array}\right) .
$$

For each matrix entry, the upper index denotes form rank while the lower index is the generation number. As mentioned above, we shall assume an acyclic background. As 
explained in $[8,29]$, this can be achieved by giving an expectation value to the field $\phi^{(0)}$, such that this vev is flat bundle isomorphism.

To construct the quantum action we add a trivial pair for each classical gauge invariance and write the non-minimal action:

$$
S_{n m}=\operatorname{Re} \int \operatorname{tr}\left[\pi_{1}^{(0)} b_{1}^{*(3)}+\pi_{1}^{\prime(0)} b_{1}^{\prime *(3)}+\pi_{1}^{(1)} b_{2}^{*(2)}+\pi_{2}^{(0)} b^{*(2)}+\ldots\right]
$$

where juxtaposition stands for the total boundary product $\bullet$ and the dots represent possible extra terms due to invariances of the gauge fixing fermion. The weighted gauge-fixing fermion has the form:

$$
\begin{aligned}
& \Psi=\operatorname{Re} \int \operatorname{tr}\left[b_{1}^{(0)} d * \phi^{(1)}+{b^{\prime}}_{1}^{(0)} d * \phi^{\prime(1)}+b_{1}^{(1)} d * \phi^{(2)}+b_{2}^{(0)} d * c_{1}^{(1)}+\check{c}_{2}^{(0)} d * b_{1}^{(1)}\right. \\
& +\Xi\left[b_{1}^{(0)} * \pi_{1}^{(0)}+b_{1}^{\prime(0)} * \pi_{1}^{\prime(0)}+b_{1}^{(1)} * \pi_{1}^{(1)}+b_{2}^{(0)} * \check{\pi}_{2}^{(0)}+\check{c}_{2}^{(0)} * \pi_{2}^{(0)}\right] .
\end{aligned}
$$

One could be tempted to write $b_{2}^{(0)} * \pi_{2}^{(0)}$ instead of the second last term. However, such a term is incompatible with the ghost number requirements for $\Psi$ and $S_{n m}$. Furthermore, the gauge invariance $b_{1}^{(1)} \rightarrow b_{1}^{(1)}+d \kappa$ requires the introduction of an extra ghost $\check{c}$. By counting the ghost numbers associated with the various fields we find that the last term in the previous equation has ghost number -1 and thus it is indeed allowed in $\Psi$. Due to the presence of these extra fields we need to add a further term to the non-minimal action:

$$
S_{\text {extra }}=\operatorname{Re} \int \operatorname{tr}\left[\check{\pi}_{2}^{(0)} \check{c}_{2}^{*}(3)\right]
$$

This satisfies the ghost number and parity constraints provided that $\check{c}$ and $\check{\pi}$ form a trivial pair. The result is the full auxiliary action $S_{a u x}=S_{n m}+S_{\text {extra }}$.

The auxiliary fields can be arranged in the following matrices:

$$
\begin{array}{ccc}
\bar{\pi}_{1}[0]=\left(\begin{array}{cc}
-\pi_{1}^{(0)} & -\pi_{1}^{(1)} \\
0 & -\pi_{1}^{\prime(0)}
\end{array}\right) & , & \bar{\phi}_{1}[0]=\left(\begin{array}{cc}
-b_{1}^{(0)} & b_{1}^{(1)} \\
0 & -b_{1}^{\prime(0)}
\end{array}\right) \\
\pi_{2}[1]=\left(\begin{array}{cc}
0 & \pi_{2}^{(0)} \\
0 & 0
\end{array}\right) & , & \bar{\phi}_{2}[0]=\left(\begin{array}{cc}
0 & b_{2}^{(0)} \\
0 & 0
\end{array}\right) \\
\phi_{2}[1]=\left(\begin{array}{cc}
0 & \check{c}_{2}^{(0)} \\
0 & 0
\end{array}\right) & , & \bar{\pi}_{2}[0]=\left(\begin{array}{cc}
0 & \check{\pi}_{2}^{(0)} \\
0 & 0
\end{array}\right) .
\end{array}
$$

This presentation allows us to write the gauge-fixing fermion and auxiliary action in the form given in the previous subsections:

$$
\begin{aligned}
\Psi= & \operatorname{Re} \int \operatorname{str}\left[\bar{\phi}_{1}[0] * d_{e} c_{e} \phi_{0}[0]+\bar{\phi}_{2}[0] * d_{e} c_{e} \phi_{1}[0]+\phi_{2}[1] * d_{e} c_{e} \bar{\phi}_{1}[0]\right. \\
& \left.+\Xi\left[\bar{\phi}_{1}[0] * c_{e} \bar{\pi}_{1}[0]+\bar{\phi}_{2}[0] * c_{e} \pi_{2}[1]+\phi_{2}[1] * c_{e} \bar{\pi}_{2}[0]\right]\right]
\end{aligned}
$$


and:

$$
\begin{aligned}
S_{a u x} & =S_{n m}+\operatorname{Re} \int \operatorname{tr}\left[\check{\pi}_{2}^{(0)} \check{c}^{*(3)}\right] \\
& =\operatorname{Re} \int \operatorname{tr}\left[\bar{\pi}_{1}[0] * \bar{\phi}_{1}^{*}[0]+\bar{\pi}_{2}[0] * \bar{\phi}_{2}^{*}[0]+\pi_{2}[1] * \phi_{2}^{*}[1]\right] .
\end{aligned}
$$

where

$$
\bar{\phi}_{1}^{*}[0]=\left(\begin{array}{cc}
-b_{1}^{*(3)} & 0 \\
-b_{1}^{*(2)} & -b_{1}^{\prime *(3)}
\end{array}\right) \quad \bar{\phi}_{2}^{*}[0]=\left(\begin{array}{cc}
0 & 0 \\
b_{2}^{*(3)} & 0
\end{array}\right) \quad \phi_{2}^{*}[1]=\left(\begin{array}{cc}
0 & 0 \\
\check{c}_{2}^{*(3)} & 0
\end{array}\right)
$$

Following standard procedure, we solve for the transformed antifields and substitute the solutions into the full action $S_{t o t}=S_{e}+S_{a u x}$. We first analyze the terms related to gauge symmetries of the two-form. The relevant terms in $S_{\text {tot }}$ are:

$$
\begin{aligned}
S_{\text {tot }} & =\int \operatorname{tr}\left[\ldots+\phi^{(0)} d \phi^{(2)}+\phi^{*(1)}\left(d c_{1}^{(1)}+\ldots\right)+c^{*}{ }_{1}^{(2)}\left(d c_{2}^{(0)}+\ldots\right)\right. \\
& \left.+\pi_{1}^{(1)} b_{1}^{*(2)}+\pi_{2}^{(0)} b_{2}^{*(3)}+\check{\pi}_{2}^{(0)} \check{c}_{2}^{*(3)}\right]+\ldots .
\end{aligned}
$$

Solving for the antifields from the gauge fixing fermion leads to the gauge-fixed action:

$$
\begin{aligned}
S_{g f} & =\operatorname{Re} \int \operatorname{tr}\left[\ldots+\phi^{(0)} d \phi^{(2)}+* d b_{1}^{(1)}\left(d_{e} c_{1}^{(1)}+\ldots\right)-* d b_{2}^{(0)}\left(d c_{2}^{(0)}+\ldots\right)\right. \\
& +\pi_{1}^{(1)}\left(\Xi * \pi_{1}^{(1)}+d * \phi^{(2)}-* d \check{c}_{2}^{(0)}\right)+\pi_{2}^{(0)}\left(\Xi * \check{\pi}_{2}^{(0)}+d * c_{1}^{(1)}\right) \\
& \left.+\check{\pi}_{2}^{(0)}\left(\Xi * \pi_{2}^{(0)}+d * b_{1}^{(1)}\right)\right]+\ldots .
\end{aligned}
$$

After eliminating the auxiliary fields and dropping total derivatives, we obtain:

$$
\begin{aligned}
S_{g f} & =\operatorname{Re} \int \operatorname{tr}\left[\ldots+\phi^{(0)} d \phi^{(2)}+* d b_{1}^{(1)}\left(d c_{1}^{(1)}+\ldots\right)-* d b_{2}^{(0)}\left(d c_{2}^{(0)}+\ldots\right)\right. \\
& \left.-\frac{1}{4 \Xi}\left(* d \check{c}_{2}^{(0)} d \check{c}_{2}^{(0)}+d * \phi^{(2)} * d * \phi^{(2)}\right)-\frac{1}{2 \Xi} d * c_{1}^{(1)} * d * b_{1}^{(1)}\right] .
\end{aligned}
$$

where we used the fact that $\pi_{2}^{(0)}$ and $\check{\pi}_{2}^{(0)}$ are anti-commuting objects. The last expression shows that the gauge-fixing conditions are actually decoupled and have the Lorentz form $d^{\dagger} \phi^{(2)}=d^{\dagger} c^{(1)}=d^{\dagger} b_{(1)}=0$. The same condition can be derived from the delta function gauge fixing fermion. Then, the auxiliary fields appear as Lagrange multipliers imposing the various gauge constraints. For example, $\pi^{(1)}$ imposes $* d * \phi^{(2)}=d \check{c}^{(0)}$. This implies that $\check{c}^{(0)}$ is a harmonic form. Since the base manifold $L$ is compact, one concludes that $\check{c}^{(0)}$ is a covariantly-constant section on $\operatorname{End}(E)$, and that $\phi^{(2)}$ also satisfies the gauge condition $d^{\dagger} \phi^{(2)}=0$. 
Some straightforward algebra leads to the complete form of the gauge-fixed action:

$$
\begin{aligned}
& S_{g f}=2 \operatorname{Re}\left\{f t \int_{L} \operatorname{tr}_{a}\left[\frac{1}{2}\left(\phi^{(1)} d \phi^{(1)}-\phi^{(2)} d \phi^{(0)}\right)+\frac{1}{3}\left(\phi^{(1)} \phi^{(1)} \phi^{(1)}+\phi^{(1)} \phi^{(2)} \phi^{(0)}\right)\right]\right. \\
& -\int_{L} \operatorname{tr}_{b}\left[\frac{1}{2}\left(\phi^{\prime(1)} d \phi^{\prime(1)}-\phi^{(0)} d \phi^{(2)}\right)+\frac{1}{3}\left(\phi^{\prime(1)} \phi^{\prime(1)} \phi^{\prime(1)}+\phi^{\prime(1)} \phi^{(0)} \phi^{(2)}\right)\right] \\
& +\int_{L} \operatorname{tr}_{a}\left[b_{1}^{(0)} d *\left(d c_{1}^{(0)}+\left[\phi^{(1)}, c_{1}^{(0)}\right]-c_{1}^{(1)} \phi^{(0)}\right)\right. \\
& +\int_{L} \operatorname{tr}_{b}\left[b_{1}^{(1)} d *\left(d c_{1}^{(1)}+\left(\phi^{(1)} c_{1}^{(1)}+\phi^{(2)} c_{1}^{\prime(0)}-c_{1}^{(0)} \phi^{(2)}+c_{1}^{(1)} \phi^{\prime(1)}\right)\right)\right. \\
& +{b^{\prime}}_{1}^{(0)} d *\left(d c_{1}^{\prime(0)}+\left[\phi^{\prime(1)}, c_{1}^{(0)}\right]-\phi^{(0)} c_{1}^{(1)}\right) \\
& \left.+b_{2}^{(0)} d *\left(c_{1}^{(0)} c_{1}^{(1)}+c_{1}^{(1)} c_{1}^{(0)}+d c_{2}^{(0)}+\phi^{(1)} c_{2}^{(0)}-c_{2}^{(0)} \phi^{\prime(1)}\right)\right] \\
& +\int_{L} t r_{a}\left(-c_{2}^{(0)} * d b_{1}^{(1)} * d b_{1}^{(0)}+c_{2}^{(0)} * d b_{1}^{\prime(0)} * d b_{1}^{(1)}\right) \\
& -\frac{1}{4 \Xi} \int_{L} \operatorname{tr}_{a}\left[d * \phi^{(1)} * d * \phi^{(1)}+d * \phi^{(2)} * d * \phi^{(2)}\right. \\
& \left.-\frac{1}{4 \Xi} \int_{L} \operatorname{tr}_{b}\left[d * \phi^{\prime(1)} * d * \phi^{(1)}+* d \check{c}_{2}^{(0)} d \check{c}_{2}^{(0)}+2 d * c_{1}^{(1)} * d * b_{1}^{(1)}\right]\right\} \text {. }
\end{aligned}
$$

This expression can be recognized as a particular case of (4.26):

$$
\begin{aligned}
S_{g f}=2 \operatorname{Re}\{ & \int_{L} \operatorname{str}\left[\frac{1}{2} \phi_{0}[0] * d_{e} \phi_{0}[0]+\frac{1}{3} \phi_{0}[0] * \phi_{0}[0] * \phi_{0}[0]\right. \\
& +c_{e} d_{e} \bar{\phi}_{1}[0] *\left(d \phi_{1}[0]+\left[\phi_{0}[0], \phi_{1}[0]\right]_{*}\right) \\
& +c_{e} d_{e} \bar{\phi}_{2}[0] *\left(d \phi_{2}[0]+\left[\phi_{0}[0], \phi_{2}[0]\right]_{*}+\left[\phi_{1}[0], \phi_{1}[0]\right]_{*}\right) \\
& +\phi_{2}[0] *\left[c_{e} d_{e} \bar{\phi}_{1}[0], c_{e} d_{e} \bar{\phi}_{1}[0]\right]_{*} \\
& \left.\left.-\frac{1}{4 \Xi}\left(\phi_{2}[1] * d_{e} c_{e} d_{e} \phi_{2}[1]+\phi_{0}[0] * c_{e} d_{e} c_{e} d_{e} c_{e} \phi_{0}[0]+2 \phi_{1}[0] * c_{e} d_{e} c_{e} d_{e} c_{e} \bar{\phi}_{1}[0]\right)\right]\right\} .
\end{aligned}
$$

The first line is the classical action, the next two lines come from terms linear in antifields, the fourth line is produced by terms quadratic in antifields, while the remaining contributions come from $S_{\text {aux }}$. We also used the fact that $d_{e}^{\dagger} \phi_{2}[1]=0$ and $d_{e}^{\dagger} \bar{\phi}_{2}[0]=0$ which follow from the explicit form of $\phi_{2}[1]$ and $\bar{\phi}_{2}[0]$.

\section{References}

[1] M. R. Douglas, D-branes, Categories and N=1 Supersymmetry, hep-th/0011017.

[2] P. S. Aspinwall, A. Lawrence, Derived Categories and Zero-Brane Stability, hepth/0104147.

[3] P. S. Aspinwall, M. R. Douglas, D-Brane Stability and Monodromy, hep-th/0110071. 
[4] C. I. Lazaroiu, Generalized complexes and string field theory, JHEP 06 (2001) 052.

[5] C. I. Lazaroiu, Unitarity, D-brane dynamics and D-brane categories, hep-th/0102183.

[6] C. I. Lazaroiu, Graded Lagrangians, exotic topological D-branes and enhanced triangulated categories, JHEP 0106 (2001) 064.

[7] D. E. Diaconescu, Enhanced D-Brane Categories from String Field Theory, hepth/0104200.

[8] C. I. Lazaroiu, R. Roiban, Holomorphic potentials for graded D-branes, hep-th/0110288.

[9] C. I. Lazaroiu, An analytic torsion for graded D-branes, hep-th/0111239.

[10] E. Witten, Chern-Simons gauge theory as a string theory, The Floer memorial volume, 637-678, Progr. Math., 133, Birkhauser, Basel, 1995, hep-th/9207094.

[11] E. Witten, Topological sigma models, Commun. Math. Phys. 118 (1988),411.

[12] E. Witten, Mirror manifolds and topological field theory, Essays on mirror manifolds, 120-158, Internat. Press, Hong Kong, 1992, hep-th/9112056.

[13] E. Witten, A note on the antibracket formalism, Mod. Phys. Lett. A5 (1990) 487.

[14] M. Henneaux, Geometric Interpretation of the Quantum Master Equation in the BRSTanti-BRST Formalism, Phys. Lett. B282 (1992) 372, hep-th/9205018.

[15] O. M. Khudaverdian, A. P. Nersessian, On the Geometry of the Batalin-Vilkovisky Formalism, Mod. Phys. Lett. A8 (1993) 2377-2386, hep-th/9303136.

[16] A. Schwarz, Geometry of Batalin-Vilkovisky quantization, Commun.Math.Phys. 155 (1993) 249-260.

[17] A. Schwarz, Semiclassical approximation in Batalin-Vilkovisky formalism, Commun.Math.Phys. 158 (1993) 373-396.

[18] A. Schwarz, Symmetry transformations in Batalin-Vilkovisky formalism, Lett.Math.Phys. 31 (1994) 299-302.

[19] A. Schwarz, Superanalogs of symplectic and contact geometry and their applications to quantum field theory, hep-th/9406120.

[20] M. Alexandrov, M. Kontsevich, A. Schwarz, O. Zaboronsky, The Geometry of the Master Equation and Topological Quantum Field Theory, Int.J.Mod.Phys. A12 (1997) 14051430, hep-th/9502010. 
[21] S. Axelrod, I. M. Singer, Chern-Simons perturbation theory, Proceedings of the XXth International Conference on Differential Geometric Methods in Theoretical Physics, Vol. 1, 2 (New York, 1991), 3-45, World Sci. Publishing, River Edge, NJ, 1992, hepth/9110056.

[22] J. Gomis, J. Paris, S. Samuel, Antibracket, Antifields and Gauge-Theory Quantization, Phys.Rept. 259 (1995) 1-145.

[23] C. Nash, D. O' Connor, BRST Quantization and the Product Formula for the RaySinger Torsion, Int.J.Mod.Phys. A10 (1995) 1779-1806, hep-th/9310038.

[24] A. Schwarz, The partition function of a degenerate quadratic functional and Ray-Singer invariants, Lett. Math. Phys, 2 (1978), 247-252.

[25] A. Schwarz, The partition function of a degenerate functional, Commun. Math. Phys, 67 (1979), 1-16.

[26] D. H. Adams, S. Sen, Partition Function of a Quadratic Functional and Semiclassical Approximation for Witten's 3-Manifold Invariant, hep-th/9503095.

[27] M. Blau, G. Thompson, Ann. Phys 205(1991) 130.

[28] J. Gegenberg, G. Kunstatter, The Partition Function for Topological Field Theories, Ann. Phys. 231 (1994) 270-289, hep-th/9304016.

[29] C. I. Lazaroiu, R. Roiban and D. Vaman, Graded Chern-Simons field theory and graded topological D-branes, hep-th/0107063.

[30] D. H. Adams, S. Sen, Phase and scaling properties of determinants arising in topological field theories, Phys. Lett. B 353 (1995) no. 4, 495-500, hep-th/9506079.

[31] J. M. Bismut and J. Lott, Flat vector bundles, direct images and higher analytic torsion, J. Amer. Math Soc 8 (1992) 291.

[32] D. Quillen, Superconnections and the Chern character, Topology, 24, No.1.(1085), 8995.

[33] C. Vafa, Brane/anti-Brane Systems and $U(N \mid M)$ Supergroup, hep-th/0101218.

[34] J. H. Horne, Skein relations and Wilson loops in Chern-Simons gauge theory, Nucl. Phys. B334 (1990) 669; Bourdeau, E.J. Mlawer, H. Riggs and H.J. Schnitzer, The quasirational fusion structure of $S U(M \mid N)$ Chern-Simons and $W-Z-W$ theories, Nucl. Phys. B372 (1992) 303; L. Rozansky and H. Saleur, Reidemeister torsion, the Alexander polynomial and $U(1,1)$ Chern-Simons theory, J. Geom. Phys. 13 (1994) 105.

[35] C. I.. Lazaroiu, String field theory and brane superpotentials, hep-th/0107162. 
[36] B. DeWitt, Supermanifolds, Cambridge Univ. Press, Cambridge, 1984.

[37] F. A. Berezin, The Method of Second Quantization, Pure and Appl. Phys. 24, Academic Press, New -York, 1966.

[38] A. Rogers, A global theory of supermanifolds, J. Math. Phys 21 (1980) 1352-1365.

[39] D. B. Ray, Reidemeister torsion and the Laplacian on lens spaces, Adv. in Math. 4 (1970), 109-126.

[40] D. B. Ray, I. M. Singer, R-torsion and the Laplacian on Riemannian manifolds, Adv. Math 7 (1971), 145-210.

[41] D. B. Ray, I. M. Singer, Analytic torsion, Proceedings of Symposia in Pure Mathematics, vol. 23, p 167, American Mathematical Society 1973. 